\title{
1 Neural mechanism of experience-dependent sensory gain control in C. elegans
} 2

3 [Author names]

4 Yosuke Ikejiri $^{1,2}$, Yuki Tanimoto ${ }^{1,5}$, Kosuke Fujita ${ }^{1,6}$, Fumie Hiramatsu ${ }^{1,7}$, Shuhei J. Yamazaki ${ }^{1}$,

5 Yuto Endo ${ }^{1,2}$, Yasushi Iwatani ${ }^{3}$, Koichi Fujimoto ${ }^{1}$, Koutarou D. Kimura ${ }^{1,2,4 *}$

8 [Author affiliations]

$9{ }^{1}$ Department of Biological Sciences, Graduate School of Science, Osaka University,

10 Toyonaka, Osaka, 560-0043, Japan

$11{ }^{2}$ Graduate School of Science, Nagoya City University, Nagoya, 467-8501, Japan

$12{ }^{3}$ Department of Science and Technology, Hirosaki University, Hirosaki, Japan

$13 \quad{ }^{4}$ RIKEN Center for Advanced Intelligence Project, Tokyo, 103-0027, Japan

14 5Present address: RIKEN Center for Brain Science, Saitama, 351-0106, Japan

$15{ }^{6}$ Present address: Department of Ophthalmology, Nagoya University Graduate School of

16 Medicine, Nagoya 466-8550, Japan

$17{ }^{7}$ Present address: Center of Advanced European Studies and Research (caesar), Self-

18 Recognition and Cannibalism, Ludwig-Erhard-Allee2, 53115 Bonn

21 [Corresponding author details]

22 Koutarou D. Kimura, Email: kokimura@nsc.nagoya-cu.ac.jp 


\section{ABSTRACT}

25 Animals' sensory systems adjust their responsiveness to environmental stimuli that vary

26 greatly in their intensity. Here we report the neural mechanism of experience-dependent

27 sensory adjustment, especially gain control, in the ASH nociceptive neurons in

28 Caenorhabditis elegans. Using calcium imaging under gradual changes in stimulus intensity,

29 we find that the ASH neurons of naive animals respond to concentration increases in a

30 repulsive odor 2-nonanone regardless of the magnitude of the concentration increase.

31 However, after preexposure to the odor, the ASH neurons exhibit significantly weak responses

32 to a small gradual increase in odor concentration while their responses to a large gradual

33 increase remain strong. Thus, preexposure changes the slope of stimulus-response

34 relationships (i.e., gain control). Behavioral analysis suggests that this gain control contributes

35 to the preexposure-dependent enhancement of odor avoidance behavior. Mathematical

36 analysis reveals that the ASH response consists of fast and slow components, and that the fast

37 component is specifically suppressed by preexposure. In addition, genetic analysis suggests

38 that $\mathrm{G}$ protein signaling is required for the fast component. Thus, our integrative study

39 demonstrates how prior experience dynamically modulates stimulus-response relationships in

40 sensory neurons, eventually leading to adaptive modulation of behavior. 


\section{INTRODUCTION}

43 Animals use their sensory organs to interpret stimuli from the external environment and to 44 effectively survive and reproduce. The intensity of these stimuli can vary by a factor of $10^{10}$, 45 although the range of neuronal activity is generally limited to a factor of $10^{2}$ (Shapley and

46 Enroth-Cugell, 1984). Thus, animals need to adjust their range of neuronal activity in

47 peripheral and central sensory systems according to stimulus intensity. Such regulation of

48 neuronal responsiveness has been reported in visual, auditory, olfactory, mechanical, and

49 nociceptive systems, in a variety of animal species ranging from invertebrates to vertebrates

50 (Carew et al., 1971; Dragoi et al., 2000; Priebe and Ferster, 2002; Root et al., 2008;

51 Ulanovsky et al., 2003; Woolf and Ma, 2007).

53 One type of neuronal response modulation is sensory gain control. Gain control refers to a

54 modulation that changes the slope of stimulus-response relationships and is different from

55 adaptation or sensitization, which decreases or increases the overall responsiveness (Figure

$56 \quad 1$-figure supplement 1). Gain control has been reported to occur in the visual, auditory and

57 somatosensory cortices of mammals and the olfactory circuit of Drosophila, and it is likely

58 conserved across taxa (Andersen et al., 1985; Anderson et al., 2017; Azimi et al., 2020;

59 Chance et al., 2002; Ohzawa et al., 1982; Olsen and Wilson, 2008). However, neuronal and/or

60 molecular mechanisms underlying gain control, as well as the effect of gain control on

61 sensory behavior, have not been sufficiently elucidated.

62

63 The nematode Caenorhabditis elegans has been widely used to study the mechanisms of

64 sensory responses because of the feasibility in analyzing neural functions at the molecular,

65 cellular, and behavioral levels. The animals respond to various sensory stimuli, such as

66 chemicals, temperature, osmolality, and mechanical stimuli, which are modulated by 
experience as learning, and the neurons and genes involved in these responses have also been identified (Bargmann, 2006; De Bono and Maricq, 2005; Ferkey et al., 2021; Sasakura and Mori, 2013). However, most of the previous sensory experiments for physiological analyses of wild-type animals employed step-wise stimuli that were not controlled gradually in a physiologically meaningful manner. In addition, sensory gain control has been shown only in a few mutant strains (Kuhara et al., 2002; Saro et al., 2020). Thus, it has not been clear whether and how sensory gain control contributes to the wild-type animals' sensory behavior, such as navigation under a chemical gradient.

C. elegans avoidance behavior to the odorant 2-nonanone is an ideal experimental paradigm to study the animals' sensory response and experience-dependent modulation. The animals avoid the odor, and this odor avoidance behavior is enhanced by preexposure to the odor for 1 hour as a type of non-associative learning (Figure 1A) (Bargmann et al., 1993; Kimura et al.,

80 2010). In previous studies, we found that the odor avoidance behavior consists of two

81 behavioral states: (1) run, a long period of straight movement, and (2) pirouette, a period of repeated short movements with frequent directional changes (Kimura et al., 2010; PierceShimomura et al., 1999). We also found that when the animals sense increases or decreases in odor concentration, it promotes pirouettes or runs, respectively (Figure 1-figure supplement 2A) (Tanimoto et al., 2017). In addition, calcium imaging of their neuronal activities with gradual changes in the odor concentration (Figure 1-figure supplement 2B) revealed that a pair of ASH nociceptive neurons and a pair of AWB olfactory neurons respond to the odor increase and decrease, respectively (Tanimoto et al., 2017). Furthermore, our pharmacological, genetic and mathematical analyses of neuronal activities have demonstrated

90 that slowly increasing intracellular calcium response to a simple gradual odor decrease in

91 AWB neurons is modeled by the leaky integration of time-differential of odor concentration 
A

$\underline{\text { assay }}$
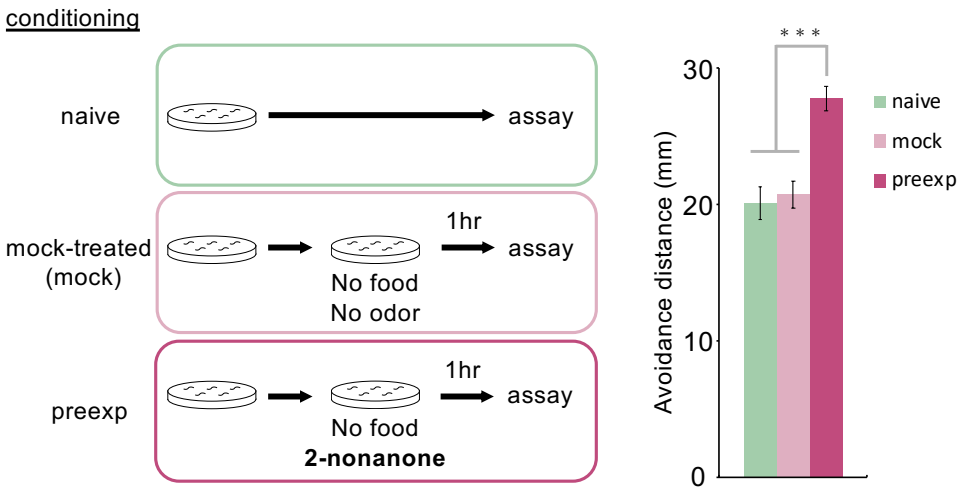

B

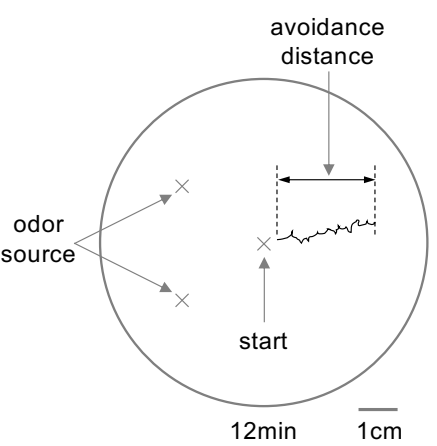

C
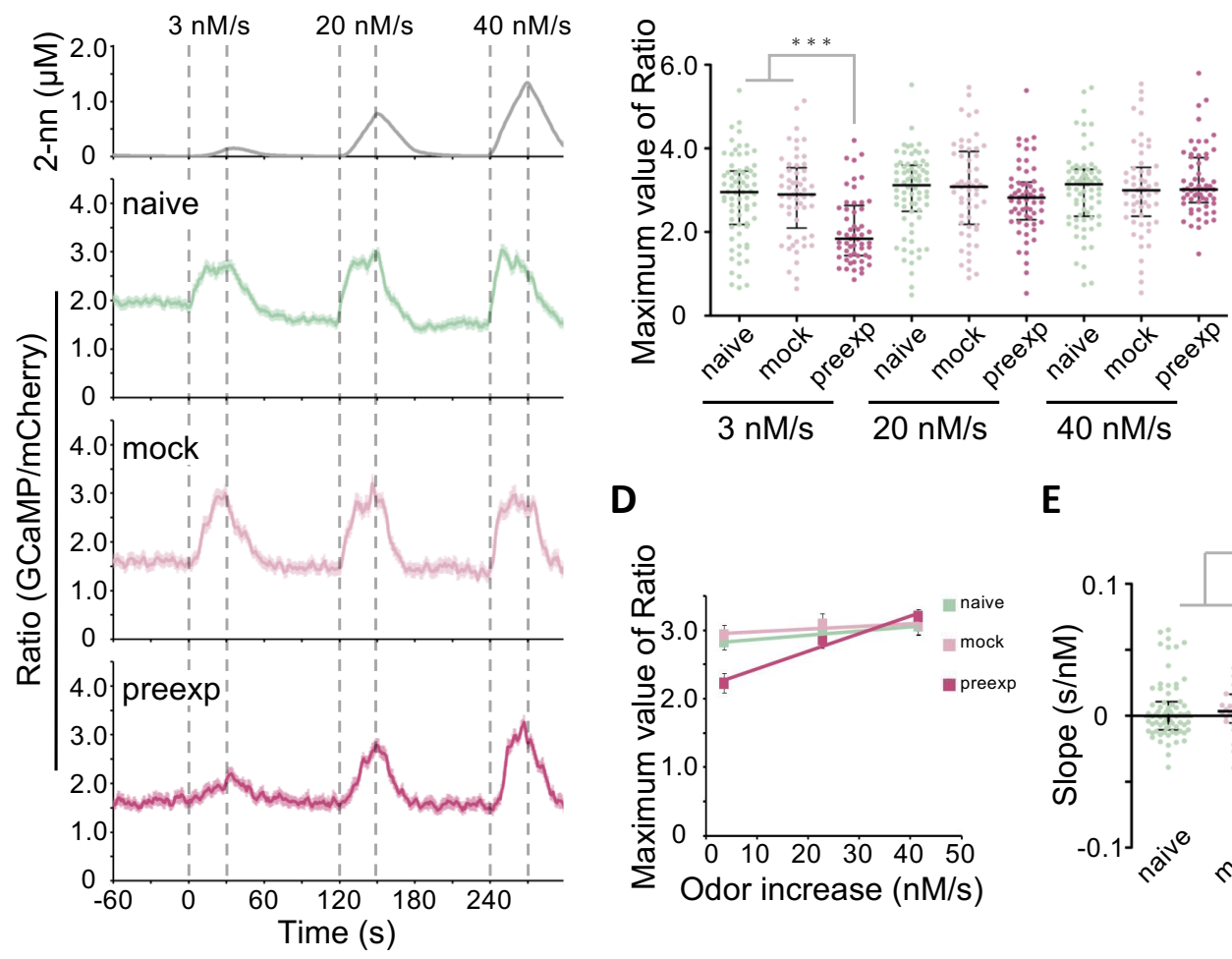

D

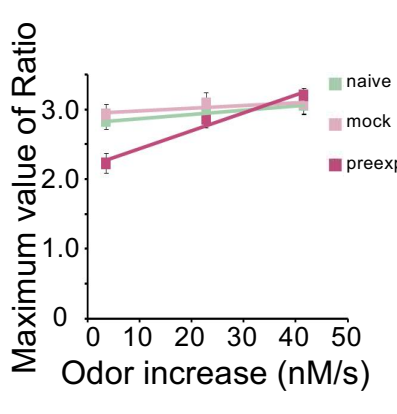

E

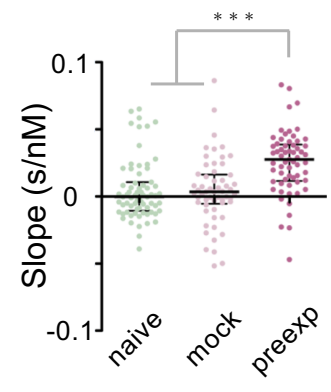

$\mathbf{F}$

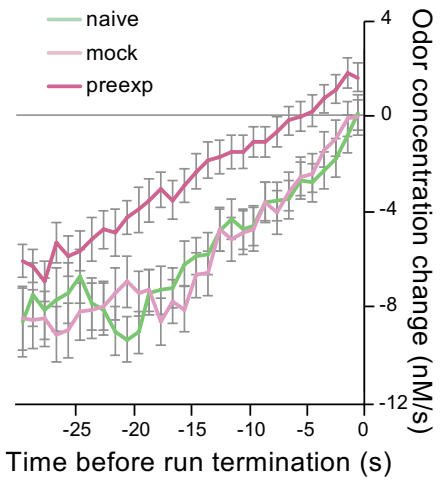

Figure 1. Preexposure to the repulsive odor 2-nonanone causes the enhancement of 2nonanone avoidance and sensory gain control in ASH neurons. A, Preexposure-dependent enhancement of odor avoidance behavior. (left) Example of an animal's trajectory tracked during 12 minutes of 2-nonanone avoidance assay. (middle) The three conditions for 2- 


\section{Figure 1 continued}

nonanone avoidance assay. (right) Result of 2-nonanone avoidance assay, where the average avoidance distance \pm standard error in each condition is shown $(n=47,45,44$ in naive, mock-treated, and preexposed conditions, respectively). Compared to control (i.e., naive and mock-treated) animals, preexposed animals exhibit significantly longer avoidance distances. ${ }^{* * *} p<0.001$ (Kruskal-Wallis test with post hoc Steel-Dwass test). B, ASH responses to three consecutive stimuli $(3,20$, and $40 \mathrm{nM} / \mathrm{s}$; top) in the three conditions. In the naive (green) and mock-treated (pale red) conditions, the responses were always large regardless of the stimulus intensity. However, in the preexposure condition (dark red), the responses were smaller for small stimuli and larger for large stimuli. The solid lines and associated shadows are the average values and their standard errors, respectively ( $\mathrm{n}=71,57,61$ in naive, mock-treated, and preexposed conditions, respectively). The black dotted lines indicate the onset and end of the odor increase phase. C, Scatter plot of the maximum values of ASH response (shown in panel B) to each stimulus. ${ }^{* * *} p<0.001$ (Kruskal-Wallis test with post hoc Steel-Dwass test). D, Correlation between the rate of odor concentration increase and the maximum value of ASH response. The mean \pm standard error of ASH maximum values in panel $\mathrm{C}$ and its linear approximation are shown. E, Scatter plot of the slope of the response. Each slope was calculated by linear approximation of maximum values of ASH responses of an animal to the three stimuli $(3,20$, and $40 \mathrm{nM} / \mathrm{s}) . * * * p<0.001$ (Kruskal-Wallis test with post hoc Steel-Dwass test). F, Time-course changes of odor concentration that the animals sensed before the initiation of a pirouette. The odor concentrations that each animal sensed 30 seconds before the initiation were ensemble-averaged. The average values \pm standard error of naive, mock-treated, and preexposed animals are shown in green, pale red and dark red, respectively ( $\mathrm{n}=50$ in all conditions). In the naive and mock-treated animals, the transition from run to pirouette on average occurred when the average value of odor concentration change became close to zero, whereas the transition occurred several seconds after the animals started sensing increases in the odor concentration in the preexposed condition. The statistical details are described in Supplemental Table 1.

Figure 1-figure supplement 1: Schematic drawing of sensory modulations.

Figure 1-figure supplement 2: Behavioral and neuronal responses of C. elegans to the repulsive odor 2-nonanone.

Figure 1-figure supplement 3: Cartoons of experience-dependent modulation of the odor avoidance behavior.

93 (see Figure 1-figure supplement 2C; for leaky integration equation, please see Materials and

94 Methods), which is mainly mediated by continuous influx of calcium ions through the L-type

95 voltage-gated calcium channel (VGCC) EGL-19 (Tanimoto et al., 2017). Pharmacological

96 analysis also suggested that the ASH response to a simple gradual odor increase (odor

97 gradient \#1) consists of an AWB-like slow component plus a fast and transient component, 
98 which has not been modeled yet (Figure 1-figure supplement 2D) (Tanimoto et al., 2017).

99 We further found that the ASH response to a small odor increase is reduced by odor

100 preexposure, indicating an experience-dependent modulation of its activity (Yamazaki et al.,

101 2019). ASH neurons are considered as a simple model for polymodal nociceptive neurons

102 evolutionarily conserved from worms to mammals (Kaplan and Horvitz, 1993). Thus,

103 analyzing the ASH response to 2-nonanone will be ideal to address questions at the levels of

104 behavior, neural activity, and molecules. For example, how do sensory neurons adapt their

105 sensitivity for behavioral responses to ever-changing environmental stimuli, which cellular

106 mechanisms support changes in response after experiencing a stimulus, and what is the

107 molecular mechanism for its implementation.

108

109 In this study, we show that sensory gain control occurs in ASH sensory neurons of C. elegans

110 for efficient odor avoidance by preexposure to 2-nonanone via specific suppression of one of

111 its activity components. Subjecting the animals to a series of gradual changes in odor

112 concentration reveals that preexposed ASH neurons become less sensitive to smaller increases

113 in odor concentration, while their responses to larger increases do not change. In other words,

114 preexposure changes the slope of the stimulus-response relationships, i.e. gain control. This

115 experience-dependent modulation of sensory activity is consistent with the changes in the

116 animals' behavioral responses: they respond less to small increases in odor concentration,

117 likely leading to more efficient odor avoidance not disturbed by a noisy and/or fluctuating

118 odor concentration change. On modeling the ASH response mathematically, we find that the

119 slow and fast components of ASH response are well fitted as the sum of the leaky integration

120 of the first- and second-order time-differentials of odor concentration, respectively. Notably,

121 the preexposure experience specifically suppresses only the fast component. Furthermore,

122 genetic analysis suggests that $\mathrm{G}$ protein signaling may regulate the fast component after 
preexposure. Thus, our results demonstrate how experience-dependent modulation of

124 stimulus-response relationships occurs and how it leads to changes in behavioral responses.

\section{RESULTS}

\section{Sensory gain control in ASH neurons caused by odor preexposure}

128 To reveal how experience changes the activities of sensory neurons under conditions of

129 physiologically meaningful odor stimuli, we investigated ASH activities before and after

130 preexposure to 2-nonanone. Our previous quantitative behavioral analysis revealed that $C$.

131 elegans senses approximately $5-20 \mathrm{nM} / \mathrm{s}$ concentration changes during 2-nonanone avoidance

132 behavior (Yamazoe-Umemoto et al., 2015). Therefore, in this experiment, we used three types

133 of stimuli in a series of odor stimulations (odor gradient \#2): (1) a minimum increase that

134 could be provided stably by our original microscope system OSB2 (3 nM/s) (Tanimoto et al.,

135 2017); (2) a maximum increase sensed during the behavioral experiment ( $20 \mathrm{nM} / \mathrm{s})$; and (3)

136 an even larger increase $(40 \mathrm{nM} / \mathrm{s})$. We measured the ASH response to these stimuli under

137 three different conditions with prior treatments: naive, mock-treated, and preexposed (Figure

138 1A).

139

140 In the naive condition, the ASH response to $3 \mathrm{nM} / \mathrm{s}$ started to increase immediately after the

141 onset of odor increase, quickly reached close to the maximum value, and the magnitude of the

142 response was sustained during the odor increase (Figure 1B). When the odor began to

143 decrease, the response also decreased, and when the odor concentration returned to zero, the

144 ASH response returned to a basal level. The ASH responses to $20 \mathrm{nM} / \mathrm{s}$ and $40 \mathrm{nM} / \mathrm{s}$ odor

145 increases were essentially similar to the response to $3 \mathrm{nM} / \mathrm{s}$ in magnitude and pattern. The

146 results of the mock-treated condition were similar to those of the naive animals. In summary,

147 the magnitude of the response was always constant in naive and mock-treated conditions, 
regardless of the magnitude of the odor increase.

150 In the preexposed condition, unlike naive and mock-treated animals, ASH did not respond

151 much to the $3 \mathrm{nM} / \mathrm{s}$ increase: its maximum value was substantially smaller than those in the

152 naive and the mock-treated animals, and the activity slowly increased and reached the maximum value at the end of the odor-increasing phase (Figure 1B). However, ASH responded strongly to the 20 and $40 \mathrm{nM} / \mathrm{s}$ increases, and the magnitude of the response increased according to the magnitude of the odor increase.

In terms of the maximum response to each odor increase, there was no significant difference in the responses to 20 and $40 \mathrm{nM} / \mathrm{s}$, but the response to $3 \mathrm{nM} / \mathrm{s}$ was significantly smaller in the preexposed animals than in the naive and mock-treated animals (Figure 1C). The linear approximation of the average responses to each odor increase did not change much in the

161 naive and mock-treated conditions, but was positive in the preexposed condition (Figure 1D).

162 Furthermore, by calculating the slope of each animal's response to the 3,20 , and $40 \mathrm{nM} / \mathrm{s}$ stimuli, we found that the slopes of the preexposed response were significantly larger than

164 those of the naive and mock-treated responses (Figure 1E). Thus, the slope of stimulusresponse relationships of ASH neurons changes because of preexposure to the odor, not

166 adaptation or sensitization, which is an overall increase or decrease of the relationships

167 without changes in its slope (Figure 1-figure supplement 1). In other words, gain control 168 occurs in ASH neurons because of preexposure to the odor.

\section{Behavioral significance of ASH gain control by preexposure}

171 Next, we investigated whether the ASH gain control after preexposure could explain the 172 enhanced 2-nonanone avoidance behavior (Kimura et al., 2010). Since 2-nonanone is a 
repulsive odor and ASH responds to the odor increase to cause turns (Tanimoto et al., 2017), a

174 simple scenario would be that ASH sensitivity increases to make the animal avoid 2-nonanone

175 sooner after preexposure. However, our results indicate that ASH was less sensitive when the

176 increase in odor concentration was small (Figure 1B).

178 To understand how changes in ASH response caused by gain control affect odor avoidance

179 behavior, we calculated time-course changes in odor concentration that each animal sensed

180 during the odor avoidance. This calculation was according to the model of 2-nonanone

181 evaporation and diffusion, which is based on the actual measurement of the local odor

182 concentration in the air phase of the plate (Tanimoto et al., 2017). We then analyzed the

183 correlations between odor concentration changes and behavioral aspects of the animals.

185 We found that the change in odor concentration upon initiation of the pirouette phase was

186 almost zero in the naive and mock-treated conditions, but was positive in the preexposed

187 condition (Figure 1F). This suggests that naive and mock-treated animals initiated pirouettes

188 in response to very small increases in odor concentration, while the preexposed animals

189 responded only to larger increases. This is consistent with our results on the ASH response,

190 which is very sensitive to a small odor increase in naive and mock-treated conditions, but less

191 sensitive in the preexposed condition (Figure 1B-E). This change can explain the enhanced

192 odor avoidance behavior by the animals after odor preexposure (Figure 1 - figure supplement

193 3) (see Discussion).

195 Modeling the experience-dependent gain control in ASH activity

196 In order to obtain quantitative insights into the mechanism of gain control caused by

197 preexposure, we performed mathematical modeling of the ASH response. In our previous 
study, the ASH response to the simple gradual odor increase (odor gradient \#1) was modeled

199 by simple time-differential of odor concentration (Tanimoto et al., 2017). Furthermore, our

200 pharmacological study suggested the ASH response to the odor gradient \#1 consists of fast

201 and slow components (Figure 1-figure supplement 2D). The slow component was modeled

202 by the leaky integration of time-differential $(d C / d t)$ of odor concentration $(C)$ like the AWB

203 response (Figure 1-figure supplement 2C). However, the simple time-differential model and

204 the leaky integration of time-differential model (Equation 1 in Materials and Methods) did not

205 reproduce the ASH responses sufficiently, especially the fast component of the naive and

206 mock-treated ASH responses to the odor gradient \#2 (Figure 2-figure supplement 1 and 2).

208 Next, we sought to model the fast component of ASH response mathematically. The fast

209 component started to increase at the onset of odor concentration increase, and soon it started

210 to decrease even when the odor concentration continued to increase (red dotted line in Figure

211 1-figure supplement 2D). This time course of the fast component can be approximated by a

212 leaky integration of the second-order time-differential $\left(d^{2} C / d t^{2}\right)$ of the odor concentration

213 (vertical gray bar and red line in " $d^{2} C / d t^{2} "$ panel in Figure 2A). To this end, we extended the

214 model, hereafter referred to as "first and second differential model", where the ASH response

215 itself is represented by a leaky integration of the sum of both the first- and second-order time-

216 differentials of the odor concentration (Figure 2A; Equation 2 in Materials and Methods).

218 We examined whether the first and second differential model could reproduce the ASH

219 responses to odor gradients \#1 and \#2. By determining the model parameters to the odor

220 gradient \#1 and each odor stimulus in odor gradient \#2 independently, in most of the

221 conditions the model reproduced the ASH activities well, including the fast component (blue

222 lines in Figure 2B). We should note that the contribution of the fast component in ASH 
A

B

$\frac{d X}{d t}=k_{1} \frac{d C}{d t}+k_{2} \frac{d^{2} C}{d t^{2}}-\frac{1}{\tau}\left(X-X_{e}\right)$
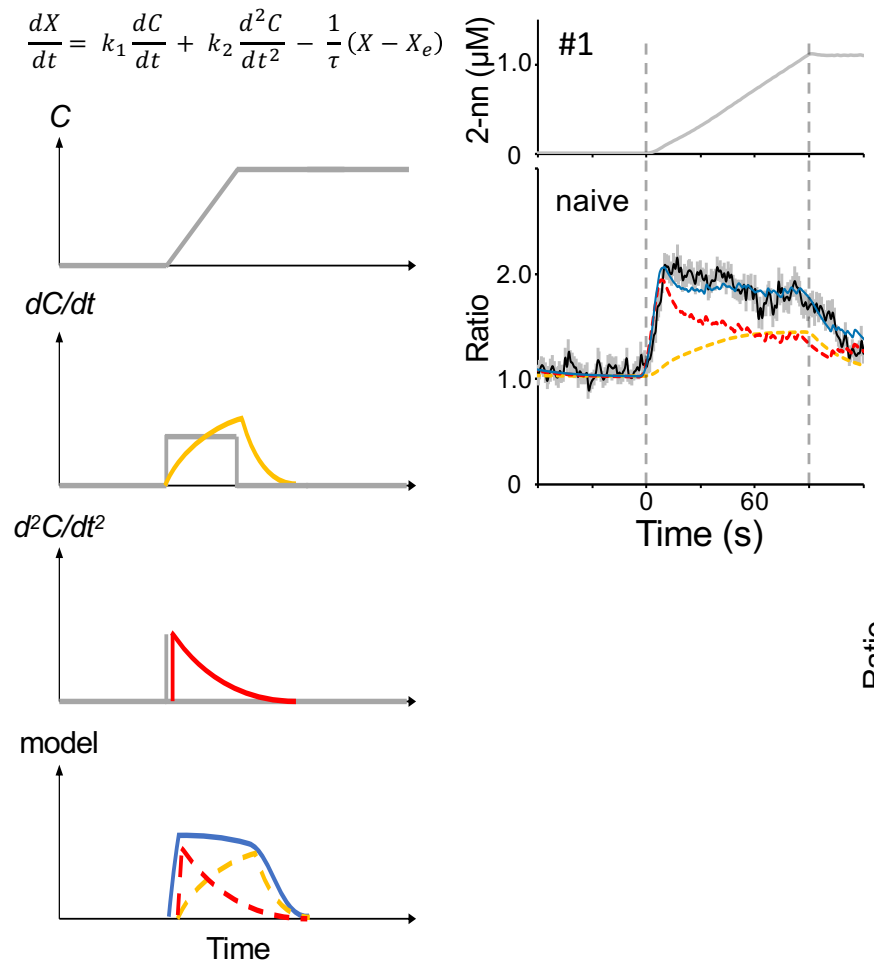

Figure 2. Mathematical model of wild-type ASH response independently fitted to a single odor stimulus. A, Schematic drawing of a mathematical model of ASH response. Equation of the first and second differential model is shown at the top. Also shown are the time course of a simple gradual odor concentration increase (top panel), first-order timedifferential of odor concentration and its leaky integration (gray rectangle and yellow line, respectively, in the second panel), second-order time-differential of the odor concentration and its leaky integration (gray vertical bar and red line, respectively, in the third panel; negative value of second-order time-differential is not calculated), and the sum of leaky integration of first- and second-order time-differentials (blue solid, yellow dashed and red dashed lines, respectively, in the bottom panel). B, The results of fitting the ASH response to the simple gradual odor increase used in the previous study (left; odor gradient \#1, $\mathrm{n}=$ 39) and independent fitting to each of three consecutive odor stimuli used in Figure 1B (right; odor gradient $\# 2, \mathrm{n}=71,57,61$ in naive, mock-treated, and preexposed conditions, respectively). The top panels exhibit each odor concentration change, and lower panels exhibit ASH responses of different conditions of animals (naive, mock-treated or preexposed) to each stimulus. The black line and the associated gray area are the average ASH responses and their standard errors, the blue line is the model, the yellow and red dashed lines are the first- and second-order components in the model, respectively. The black dotted line indicates the onset and end of the odor increase phase. 
Figure 2-figure supplement 1: Independent fitting of the wild-type ASH response to the odor stimuli with the original simple time-differential model.

Figure 2-figure supplement 2: Independent fitting of the wild-type ASH response to the odor stimuli with the leaky integration of first-order time-differential model.

Figure 2-figure supplement 3: Independent fitting of the wild-type ASH response to the odor stimuli with the leaky integration of second-order time-differential model.

226 activities was significantly lower in preexposed than in naive and mock-treated conditions

227 (red lines in right panels in Figure 2B), indicating that the fast component was specifically suppressed by the preexposure (see below).

229

230 To test the optimality of the first and second differential model, we compared the goodness of

231 fit among the first and second differential model and three other models: the original simple

232 time-differential model, and leaky integration of only first- or second-order time-differential

233 models (Figure 2-figure supplement 1, 2 and 3, respectively). By calculating the Bayesian

234 Information Criterion (BIC) (Schwarz, 1978) for each model, we found that the first and

235 second differential model had the best or close-to-the-best goodness of fit in all naive and

236 mock-treated conditions (Table 1), demonstrating the optimality of the model for these

237 conditions. In the preexposed condition, the leaky integration of first-order time-differential

238 model had the best fit, consistently with the suppression of the fast (second-order time-

239 differential) component in ASH activity (Figure 2B right).

241 The contribution of the first and second differential exhibited a remarkable difference for

242 every odor gradient \#2 (Figure 2B right). For example, in naive and mock-treated conditions,

243 the fast component became smaller when odor concentration increased more rapidly (compare

244 the response to the first odor stimulus to the ones to the second and third odor stimuli).

245 Consistently, when these parameters were uniquely determined for all the odor stimuli, the 
model did not accurately reproduce the ASH responses to odor gradient \#2 (Figure 3-figure

247 supplement 1). These results suggest that the parameters depend on some aspects of the

248 stimuli such as the odor concentration and/or its changing velocity.

250 To test whether and how the parameters depend on aspects of stimuli, we plotted the relationships between the parameters and multiple aspects of the odor stimuli. We found that each parameter approximately follows a logarithmic function of the stimulus intensity (Figure 3-figure supplement 2). Interestingly, in the well-fitted cases (red rectangles in Figure 3-

254 figure supplement 2), this function of the slow component $\left(k_{l}\right)$ and the leaky part $(1 / \tau)$ were similar among naive, mock-treated and preexposed conditions, although that of the fast component $\left(k_{2}\right)$ was much lower for preexposed than for naive and mock-treated conditions.

When we further introduced this stimulus intensity dependency into the first and second differential model (Equation 3 in Materials and Methods), the model unifyingly reproduced the ASH responses to odor gradients \#1 and \#2 as well as their gain control-dependent changes (Figure 3A and B). These results suggest that the contributions of fast and slow components and the leak of ASH response depend on the logarithmic function of the odor stimulus intensity and that the fast component is further suppressed after odor preexposure for the gain control. The differences in the relationships between stimuli aspects and each

264 parameter may reflect the regulatory mechanisms of fast and slow components and the leak activity (see Discussion).

\section{Genetic analysis of the ASH response}

268 Finally, we tried to identify the genes responsible for the fast component of ASH responses.

269 Specifically we looked for mutants that exhibit the slow component-like response in naive or 270 mock-treated (i.e., control) conditions. 
A
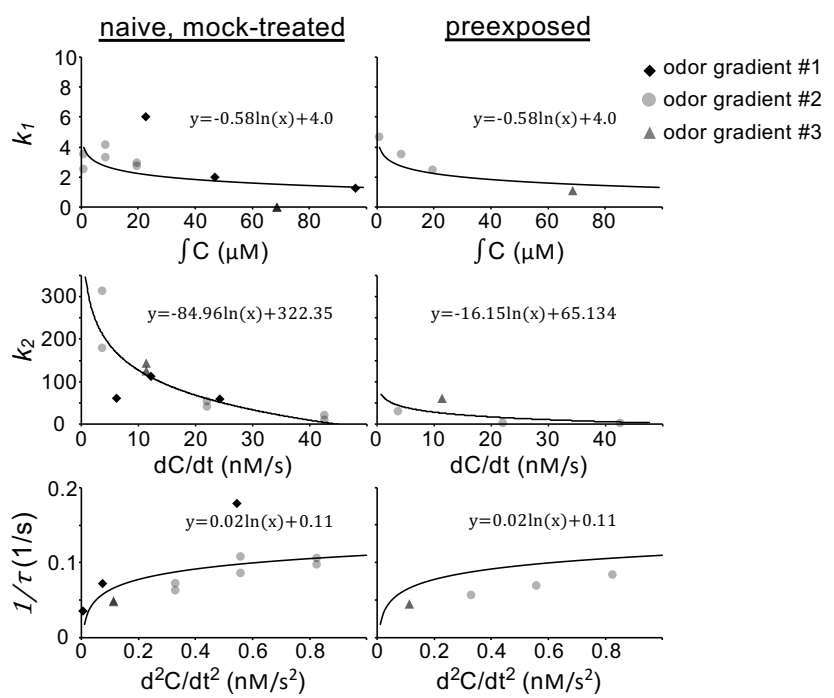

\section{B}
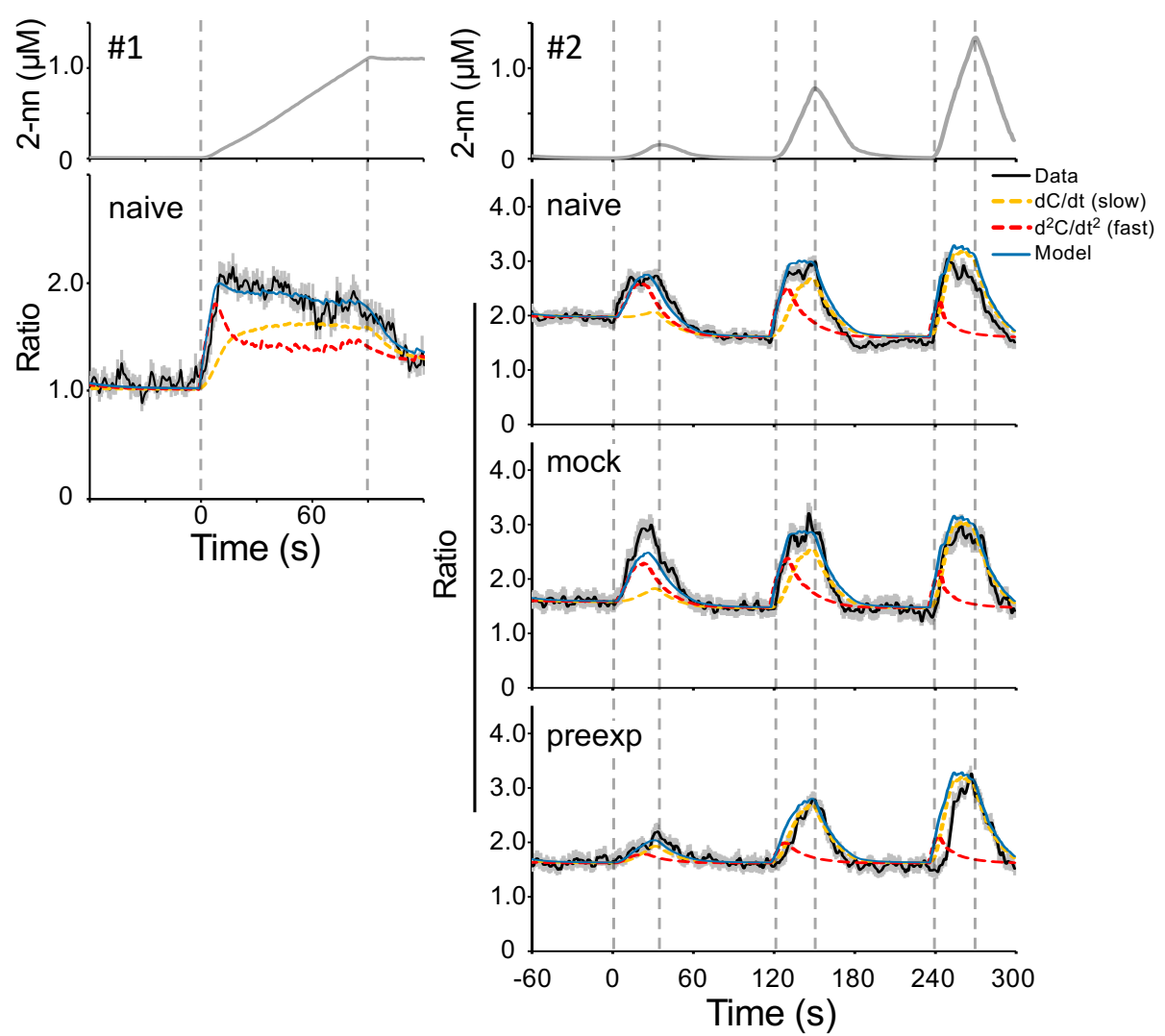

Figure 3. Model of wild-type ASH response fitted to the odor gradient \#1 and \#2 with stimulus-dependent parameters. A, Scatter plots used for the first and second differential model with stimulus-dependent parameters. Only the parameter of the second-order timedifferential, $k_{2}$, is substantially different between naive, and mock-treated versus preexposed conditions. Black rhombuses, light gray circles, and dark gray triangles represent each ASH response to odor gradient \#1, \#2, and \#3, respectively. Odor gradient \#3 was used for the genetic analysis (Figure 4). B, ASH response and its model to a simple Figure 3 continued on next page 


\section{Figure 3 continued}

odor increase (left; odor gradient \#1, $\mathrm{n}=39$ ) and to the three consecutive stimuli (right; odor gradient $\# 2, \mathrm{n}=71,57,61$ in naive, mock-treated, and preexposed conditions, respectively). The black line and the associated gray area are the average ASH responses and their standard errors, the blue line is the model, the yellow and red dashed lines are the first- and second-order component in the model, respectively. The black dotted line indicates the onset and end of the odor increase phase.

Figure 3-figure supplement 1: Fitting of the model with constant coefficients for whole naive ASH response to the odor gradient $\# 2$.

Figure 3-figure supplement 2: Scatter plots of the relationship between each input and parameter.

274 We first analyzed mutants of cation channels. osm-9 and ocr-2 are the homologs of TRPV

cation channels and known to function in ASH neurons for depolarization caused by sensory

stimuli (Colbert et al., 1997; Tobin et al., 2002) although their physiological role in 2-

nonanone sensation has not been revealed. osm-9 ocr-2 double mutants exhibited substantially no response (Figure 4A), suggesting that they are also required for sensory depolarization caused by 2-nonanone. The L-type VGCC subunit $\alpha 1$ EGL-19 is responsible for the slow component, although loss-of-function mutations in N- and T- types of VGCC subunit $\alpha 1$, unc2 and $c c a-1$ respectively, did not affect ASH response to the odor (Tanimoto et al., 2017). In this study we tested the homologs of a VGCC auxiliary subunit $\alpha 2 \delta 2$ and a sodium leak channel, tag-180 and unc-77, respectively (Lainé et al., 2011; Yeh et al., 2008). Both of the mutant strains exhibited wild-type like responses that were properly fitted with the ASH model (Figure 4A), indicating that these genes are not involved in the ASH response either.

287 We then analyzed other candidates that have been known to be involved in modulation of sensory neuronal activity in C. elegans: egl-4 (cyclic GMP-dependent protein kinase: PKG), and rgs-3 (regulator of G protein signaling: RGS) (Ferkey et al., 2007; L'Etoile et al., 2002). The ASH responses of egl-4 and $\mathrm{rgs}_{\mathrm{s}} 3$ mutants in the control condition were substantially

291 suppressed (black lines in Figure 4A). Remarkably, the ASH responses of egl-4 and rgs-3 
A
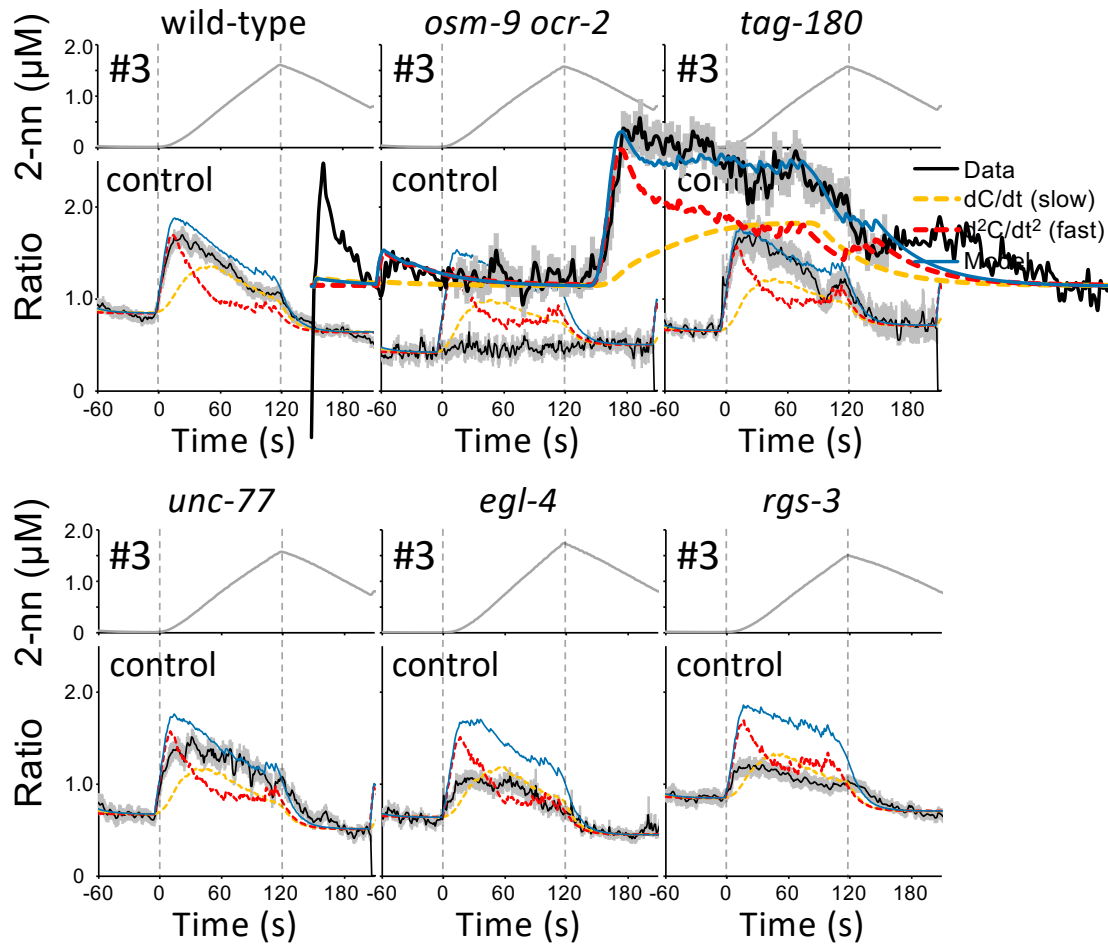

B

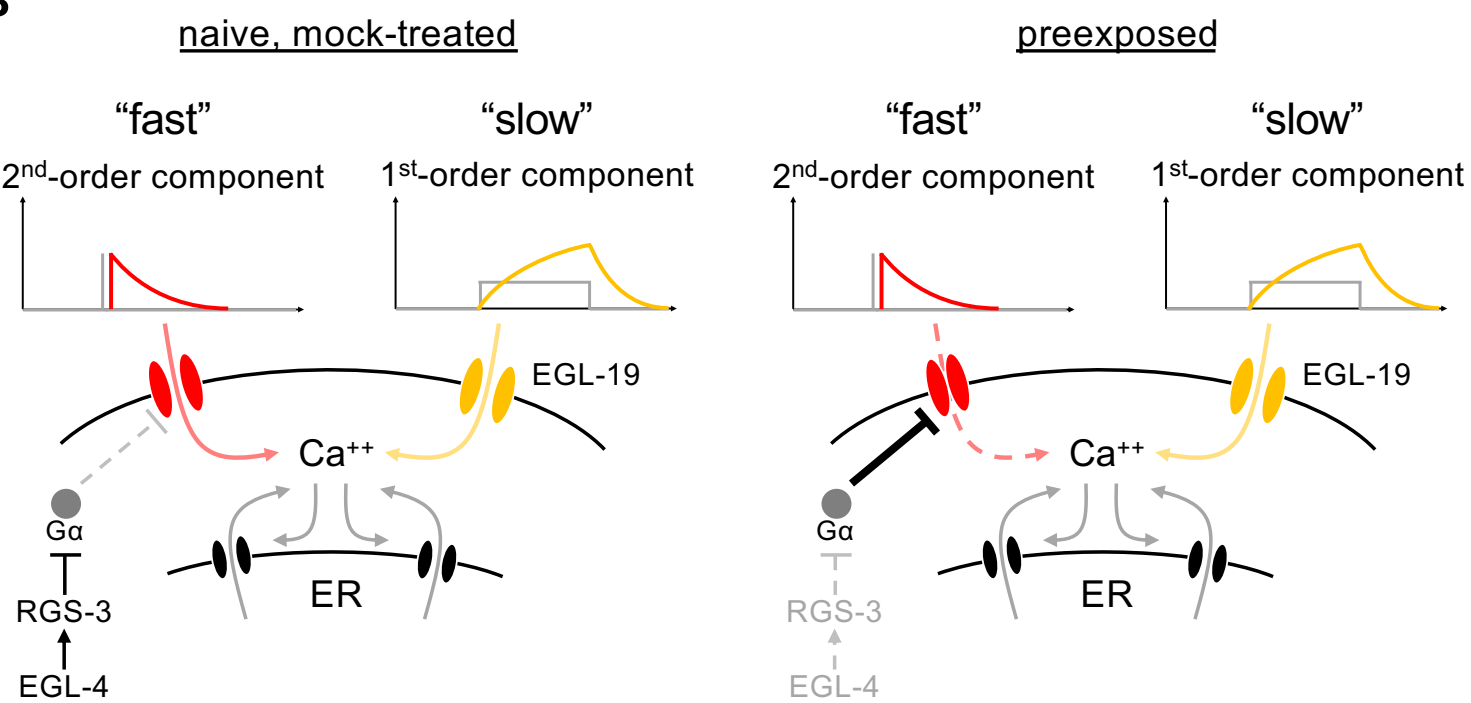

Figure 4. Genetic analysis of the ASH gain control. A, ASH response in wild-type and mutant animals in control condition ( $\mathrm{n}=51$ in wild-type; $\mathrm{n}=7$ in osm-9 ocr- $2 ; \mathrm{n}=15$ in tag-180; $\mathrm{n}=16$ in unc-77; $\mathrm{n}=46$ in egl-4; $\mathrm{n}=29$ in $\mathrm{rgs}-3$ ) to a longer and larger continuous odor increase (up to $\sim 2 \mu \mathrm{M}$ within $120 \mathrm{~s}$; odor gradient \#3). We used this odor stimulus with the idea that even milder mutant phenotypes would be observed clearly with a longer and larger stimulus. The black line and the associated gray area are the average ASH responses and their standard errors. The model parameters to fit for the wild-type Figure 4 continued on next page 


\section{Figure 4 continued}

response in Figure 3 were used, and overlayed with each of the ASH responses. The black dotted line indicates the onset and end of the odor increase phase. B, A proposed molecular model of ASH response. ASH response is mainly mediated by transiently active calcium channels (red) and persistently active L-type VGCCs (yellow). G $\alpha$ protein activity can suppress the transiently active calcium channels, and this $\mathrm{G} \alpha$ protein activity is inhibited by PKG-RGS pathway in naive/mock-treated conditions. Gain control is caused only by suppression of the transiently active calcium channels via the $\mathrm{G} \alpha$ signaling. The calcium influx from the plasma membrane is further increased by the calcium-induced calcium release through IP3 receptor and ryanodine receptor on ER membrane (Tanimoto et al., 2017).

mutants were best fitted with the first-order (i.e., slow) only model (yellow lines in Figure 4A

and Table 2), suggesting that the fast component of ASH activity requires these gene products.

It has been reported that EGL-4 phosphorylates RGS-3, and loss-of-function mutations in either of the genes increases ASH neuronal and ASH-mediated behavioral responses to other repulsive stimuli (Krzyzanowski et al., 2013). Therefore, this PKG-RGS pathway may regulate the fast component of the ASH response in a different manner from this previous report (see Discussion).

\section{DISCUSSION}

\section{2-nonanone avoidance enhancement by gain control in ASH sensory neurons}

304 In this study, we demonstrated that the responsiveness of the ASH sensory neurons of $C$. elegans to 2-nonanone exhibits gain control after preexposure to the odor. In naive and mocktreated conditions, ASH always exhibits a large response regardless of the rate of odor increase, whereas in the preexposed condition, the response to a small gradual increase became smaller and the response to a large gradual increase remains unchanged (Figure 1B-

309 E). This suggests that gain control to change the slope of stimulus-response relationships is caused by experiencing odor preexposure. 
312 Based on previous findings (Tanimoto et al., 2017) and the results of this study (Figure 1F),

313 we consider that this sensory gain control contributes to the ability of C. elegans to efficiently

314 navigate down the gradient of repulsive odor. The animals in the 2-nonanone avoidance assay

315 generally move away from the odor source and sense decreases in the odor concentration.

316 However, their movements away from the odor source are frequently interrupted because of

317 stochastic occurrence of pirouettes and/or of small increases in odor concentration (Yamazoe-

318 Umemoto et al., 2015). This increase of odor concentration could be caused by (1) the

319 direction of the animals' movement straying from the ideal, (2) small fluctuations in the odor

320 gradient, and/or (3) small periodic changes in the sensed odor concentration caused by the

321 animals' sinusoidal head swing. In the naive and mock-treated conditions, the animals initiate

322 pirouettes when they experience even a small increase in odor concentration (Figure 1-

323 figure supplement 3, top left). However, in the preexposed condition, the animals do not

324 respond to small increases (Figure 1-figure supplement 3, top right), which results in a

325 longer avoidance distance traveled in the same period. They still initiate pirouettes when they

326 sense larger increases (Figure 1-figure supplement 3, bottom panels). Thus, experience-

327 dependent changes in ASH response due to gain control likely contribute the enhanced odor

328 avoidance behavior, which could also be effective in a real-world environment infused with

329 noise.

330

331 To our knowledge, this is the first report of sensory gain control in wild-type C. elegans.

332 There are multiple examples of behavioral changes in C. elegans due to activity changes in

333 sensory neurons that are affected by repeated stimuli, different types of stimuli, or the feeding

334 state (Chew et al., 2018; Ezcurra et al., 2016; Larsch et al., 2015). However, most of the

335 response changes in wild-type animals are caused by adaptation or sensitization but not gain

336 control. It is possible that the animals might have developed a special mechanism against this 
odorant to effectively avoid danger because it is one of the two major volatile compounds produced by $P$. aeruginosa, a bacterial species that is pathogenic to C. elegans (Labows et al., 1980; Tan et al., 1999; Tan and Ausubel, 2000).

\section{Estimating the neural mechanism of gain control by mathematical modeling}

342 To obtain quantitative insights into the mechanism of sensory gain control, we performed mathematical modeling. Indeed, mathematical modeling has successfully revealed essential

344 features of the sensory neuronal responses of the animals (Ikeda et al., 2021; Itskovits et al.,

345 2018; Kato et al., 2014; Tanimoto et al., 2017). Our previous pharmacological analysis

346 revealed that ASH activity is composed of fast and slow components, and we showed in this

347 study that the fast component can be approximated by a leaky integration of the second-order

348 time-differential of the odor concentration (Figure 2). Even independent of the

349 pharmacological result, the ASH responses in wild-type animals exhibit overshoot in response 350 to a long-lasting and linearly increasing odor concentration (odor gradient \#1 in Figure 1351 figure supplement 2D and Figure 2B, and odor gradient \#3 in Figure 4A). More precisely, the response first increases rapidly, and then slowly decreases to a non-zero constant value. The leaky integration of the first-order time-differential of the odor concentration generates a sustained response that converges to a non-zero constant value. The leaky integration of the second-order time-differential generates a rapidly increasing and subsequently decreasing response. Thus, both the pharmacological analysis as well as the analysis of shapes of ASH response in time domain suggest that the ASH response can be modeled with a leaky integration of the sum of the first- and second-order time-differentials of the odor concentration, and that these two terms well describe essential characteristics of ASH neurons. Indeed, the model had the best goodness of fit in most naive and mock-treated conditions in terms of BIC (Table 1). It should be noted, however, that we only tested the 
362 leaky integration of the second-order time-differential equation to the constantly increasing

363 odor gradients, and the equation may not be applicable to ASH responses to other types of

364 odor stimuli; in other words, the fast component could be approximated with another equation

365 such as an alpha function (Rall, 1967). Nevertheless, the leaky integration of the sum of the

366 first- and second-order time-differential equation with stimulus-dependent parameters nicely

367 reproduced the ASH responses, and provided us an important biological insight (see next

368 section). Some details still do not match, suggesting the involvement of other factors in the

369 ASH response.

370

371 Remarkably, our model indicates that the preexposure-dependent changes in ASH response

372 can be caused only by the modulation of the fast component $\left(k_{2}\right)$ rather than the modulation of

373 overall ASH response, although the ASH response does exhibit changes in multiple aspects,

374 such as its speed, magnitude, and small increase/decrease when it reaches closes to the

375 maximum value (odor gradient \#2 in Figure 2B). It also explains why ASH responses to a

376 large odor increase are not substantially affected. This result suggests that a specific sensory

377 signaling molecular pathway that suppresses the fast component in ASH neurons is regulated

378 by preexposure to cause experience-dependent modulation of behavior, i.e., learning.

380 Relationships between the mathematical model and molecular mechanisms of ASH

\section{1 response}

382 We have previously shown that AWB responses are mediated by EGL-19, an $\alpha 1$ subunit of L-

383 type VGCC, which possesses a long-lasting channel opening property, and that a $\mathrm{G} \alpha_{\mathrm{o}}$

384 homolog, GOA-1, is required for the first-order time-differential of odor concentration. These

385 results suggest that the constant decrease in 2-nonanone concentration is time-differentiated at

386 the sensory ending by $\mathrm{G}$ protein signaling and causes constant depolarization, leading to 
EGL-19 opening and a continuous influx of calcium into the cells. The slow component in ASH neurons is also mediated by EGL-19 (Tanimoto et al., 2017).

In contrast, the fast component in ASH neurons is likely caused by a transient calcium influx at the onset of odor concentration increase, which ends rapidly (Figures 2A and 4B left). One candidate gene product for this was the T-type VGCC ("T" for transient opening) (Nowycky et al., 1985). However, the loss-of-function mutant of the $c c a-1$ gene, the sole homolog of Ttype VGCC in C. elegans (Steger et al., 2005), showed wild-type-like ASH responses (Tanimoto et al., 2017). In addition, the loss-of-function mutants of N-type homolog unc-2 (Tanimoto et al., 2017) and of the homologs of VGCC auxiliary subunit $\alpha 2 \delta 2 \operatorname{tag}-180$ (Figure 4A) also exhibited a wild-type-like response, suggesting that some other calcium channel(s) may be involved in the fast component.

Still, we found that PKG EGL-4 and RGS RGS-3 possibly modulate the fast component. RGS is known to inhibit G protein signaling to modulate the magnitude and/or time-course of neuronal responses in C. elegans and mammals (Cao et al., 2012; Krzyzanowski et al., 2013; Lur and Higley, 2015). In addition, PKG phosphorylates RGS to increase its activity in $C$. elegans and mammals (Huang et al., 2007; Krzyzanowski et al., 2013). Because the effects of PKG-RGS activation on neuronal responses are different between the previous reports and our result, we consider that these gene products may inhibit G protein activity which suppresses the ASH response. In other words, in naive/mock-treated ASH neurons, the PKGRGS pathway inhibits G protein activity that suppresses the ion channel that mediates the fast component, and preexposure suppresses the PKG-RGS pathway, leading to activation of $\mathrm{G}$

410 protein and to inhibition of the fast component (Figure 4B). 
412 While genetic analysis itself is static (i.e., it would not provide us information related to time-

413 course changes of the gene product activities), the combination with physiological analysis

414 and mathematical modeling provides insights as to how these gene products affect neural

415 activities dynamically. For example, the characteristics of ASH neurons in wild-type animals

416 are not clear except for the preexposure-dependent gain control aspect, although a simple

417 "first and second differential model" can explain multiple aspects of the differences, such as

418 the quick rise at the onset of the odor stimulus, the gradual decrease during a long-lasting

419 odor increase, the slow decay during odor decrease (not reproduced by the original simple

420 time-differential model) as well as the gain control (Figures 2 and 3B). Furthermore, the

421 stimulus-dependent parameters we used in the model may reflect the opening probabilities of

422 the channels for the fast and slow responses, which change according to the intensity of the

423 stimulus. For example, $k_{l}$ reduces according to the accumulation of odor concentration

424 (Figure 3A), which may indicate habituation due to the sustained stimulation from the odor.

425 Further, $k_{2}$ reduces according to the average of $d C / d t$, suggesting that the depolarization level

426 may affect the opening probability because the odor concentration is time-differentiated at the

427 sensory ending and it reflects the depolarization level (Tanimoto et al., 2017). Lastly, $1 / \tau$

428 increases according to the second-order time-differential of odor concentration, which may

429 reflect a change in leakage level at the stimulus onset. Those insights could have not been

430 obtained without the model.

432 In summary, our results demonstrate that gain control occurs in ASH sensory neurons after 433 preexposure to the repulsive odor, and mathematical analysis suggests that this gain control is 434 caused by suppression of the fast component, which could be caused by transient cation influx 435 at the onset of odor concentration increase. This gain control leads to efficient avoidance 436 behavior that allows the animal to ignore slight increases in odor concentration while moving 
437 down the odor gradient, thereby enhancing the avoidance distance. In more complex animals,

438 such as mice and Drosophila, neurotransmitters such as serotonin or GABA are involved in

439 gain control in peripheral and central sensory systems (Azimi et al., 2020; Olsen and Wilson,

440 2008) but little is known about their detailed molecular mechanism. This study may

441 contribute to our understanding of intracellular mechanisms surrounding sensory gain control

442 in animals.

443

444 MATERIALS AND METHODS

Key Resources Table

\begin{tabular}{|c|c|c|c|c|}
\hline $\begin{array}{l}\text { Reagent type } \\
\text { (species) or } \\
\text { resource }\end{array}$ & Designation & $\begin{array}{l}\text { Source or } \\
\text { reference }\end{array}$ & Identifiers & Additional information \\
\hline $\begin{array}{l}\text { strain, strain } \\
\text { background } \\
\text { (C. elegans) }\end{array}$ & JC2628 & CGC & N/A & osm-9(ky10) ocr-2(ak47) \\
\hline $\begin{array}{l}\text { strain, strain } \\
\text { background } \\
\text { (C. elegans) }\end{array}$ & RB1780 & CGC & $\begin{array}{l}\text { RRID:WB- } \\
\text { STRAIN: } \\
\text { WBStrain0 } \\
\text { 0032471) }\end{array}$ & $r g s-3(o k 2288)$ \\
\hline $\begin{array}{l}\text { strain, strain } \\
\text { background } \\
\text { (C. elegans) }\end{array}$ & MT1073 & CGC & $\begin{array}{l}\text { RRID:WB- } \\
\text { STRAIN: } \\
\text { WBStrain0 } \\
0026779\end{array}$ & egl-4(n478) \\
\hline $\begin{array}{l}\text { strain, strain } \\
\text { background } \\
\text { (C. elegans) }\end{array}$ & DR1089 & CGC & $\begin{array}{l}\text { RRID:WB- } \\
\text { STRAIN: } \\
\text { WBStrain0 } \\
0006352\end{array}$ & unc-77(e625) \\
\hline $\begin{array}{l}\text { strain, strain } \\
\text { background } \\
\text { (C. elegans) }\end{array}$ & VC550 & CGC & $\begin{array}{l}\text { RRID:WB- } \\
\text { STRAIN: } \\
\text { WBStrain0 } \\
0035865\end{array}$ & tag-180(ok779) \\
\hline $\begin{array}{l}\text { strain, strain } \\
\text { background } \\
\text { (C. elegans) }\end{array}$ & KDK70034 & $\begin{array}{l}\text { (Tanimoto } \\
\text { et al., 2017) }\end{array}$ & N/A & $\begin{array}{l}\text { N2;oskEx70034 [sra- } \\
6 p:: G C a M p 3, \text { sra- } \\
6 p:: \text { Cherry, lin- } 44 p:: \text { GFP, } \\
\text { PvuII-cut N2 genomic DNA] }\end{array}$ \\
\hline $\begin{array}{l}\text { strain, strain } \\
\text { background } \\
\text { (C. elegans) }\end{array}$ & KDK70072 & $\begin{array}{l}\text { (Tanimoto } \\
\text { et al., 2017) }\end{array}$ & N/A & $\begin{array}{l}\text { N2;oskEx70072 [sra- } \\
6 p:: G C a M p 3, \text { sra- } \\
6 p:: m C h e r r y, ~ l i n-44 p:: G F P, \\
\text { PvuII-cut N2 genomic DNA }]\end{array}$ \\
\hline
\end{tabular}




\begin{tabular}{|c|c|c|c|c|}
\hline $\begin{array}{l}\text { strain, strain } \\
\text { background } \\
\text { (C. elegans) }\end{array}$ & KDK70349 & This study & N/A & 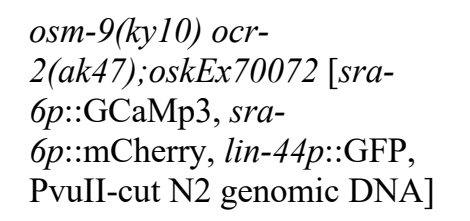 \\
\hline $\begin{array}{l}\text { strain, strain } \\
\text { background } \\
\text { (C. elegans) }\end{array}$ & KDK34005 & This study & N/A & $\begin{array}{l}\text { rgs-3(ok2288);oskEx70072 } \\
\text { [sra-6p::GCaMp3, sra- } \\
\text { 6p::mCherry, lin-44p::GFP, } \\
\text { PvuII-cut N2 genomic DNA] }\end{array}$ \\
\hline $\begin{array}{l}\text { strain, strain } \\
\text { background } \\
\text { (C. elegans) }\end{array}$ & KDK34009 & This study & N/A & $\begin{array}{l}\text { egl-4(n478);oskEx70072 [sra- } \\
6 p:: \mathrm{GCaMp} 3, \text { sra- } \\
6 p:: \text { mCherry, lin-44p::GFP, } \\
\text { PvuII-cut N2 genomic DNA] }\end{array}$ \\
\hline $\begin{array}{l}\text { strain, strain } \\
\text { background } \\
\text { (C. elegans) }\end{array}$ & KDK70342 & This study & N/A & $\begin{array}{l}\text { unc-77(e625);oskEx } 70072 \\
\text { [sra-6p::GCaMp3, sra- } \\
\text { 6p::mCherry, lin-44p::GFP, } \\
\text { PvuII-cut N2 genomic DNA] }\end{array}$ \\
\hline $\begin{array}{l}\text { strain, strain } \\
\text { background } \\
\text { (C. elegans) }\end{array}$ & KDK70347 & This study & N/A & $\begin{array}{l}\text { tag-180(ok779); oskEx } 70072 \\
{[\text { sra-6p::GCaMp3, sra- }} \\
6 p:: \text { mCherry, lin-44p::GFP, } \\
\text { PvulI-cut N2 genomic DNA] }\end{array}$ \\
\hline $\begin{array}{l}\text { recombinant } \\
\text { DNA reagent }\end{array}$ & pMIY69 & $\begin{array}{l}\text { (Tanimoto } \\
\text { et al., 2017) }\end{array}$ & N/A & $\begin{array}{l}\text { mCherry (Clontech) was PCR } \\
\text { amplified and inserted into NheI } \\
\text { (vector) / SpeI (insert) and } \\
\text { EcoRV sites of pPD49.26 (a gift } \\
\text { from A. Fire). }\end{array}$ \\
\hline $\begin{array}{l}\text { recombinant } \\
\text { DNA reagent }\end{array}$ & pKFU177 & $\begin{array}{l}\text { (Tanimoto } \\
\text { et al., 2017) }\end{array}$ & N/A & $\begin{array}{l}\text { sra- } 6 \text { promoter was amplified } \\
\text { from N2 genome by PCR and } \\
\text { inserted into HindIII and XbaI } \\
\text { sites of pMIY } 69 .\end{array}$ \\
\hline $\begin{array}{l}\text { recombinant } \\
\text { DNA reagent }\end{array}$ & pYFU107 & $\begin{array}{l}\text { (Tanimoto } \\
\text { et al., 2017) }\end{array}$ & N/A & $\begin{array}{l}\text { GCaMP3 (AddGene) was PCR } \\
\text { amplified and inserted into } \\
\text { NheI (vector) / SpeI (insert) } \\
\text { and EcoRV sites of pPD49.26. } \\
\text { sra-6 promoter from pKFU177 } \\
\text { was inserted into Xbal site of } \\
\text { the plasmid. }\end{array}$ \\
\hline
\end{tabular}

446 Strains.

447 The techniques we used to culture and handle C. elegans were essentially as described

448 previously (Brenner, 1974). C. elegans wild-type Bristol strain N2, JC2628 osm-9(ky10) ocr-

449 2(ak47), RB1780 rgs-3(ok2288), MT1073 egl-4(n478), DR1089 unc-77(e625), and VC550

450 tag-180(ok779), were obtained from the Caenorhabditis Genetics Center (University of

451 Minnesota, USA). In all the behavioral and physiological experiments, we used young adult 
hermaphrodites.

453

\section{Calcium imaging by OSB2 system.}

455 Calcium imaging of ASH sensory neurons was performed as previously reported (Tanimoto et 456 al., 2017; Tanimoto and Kimura, 2021). In brief, transgenic lines expressing GCaMP3 (Tian et al., 2009) and mCherry (Shaner et al., 2004) in ASH (20 ng/ $\mu 1$ of $s r a-6 p:: G C a M P 3,20 \mathrm{ng} / \mu 1$ of $s r a-6 p:: m C h e r r y, 10 \mathrm{ng} / \mu 1$ of lin-44p::GFP, $50 \mathrm{ng} / \mu 1$ of PvuII-cut N2 genomic DNA) were placed on NGM plates and observed under our original microscope system, OSB2. To measure the neural activity of multiple animals in a single observation, the transgenic animals were immobilized using levamisole, an agonist to the acetylcholine receptor (Lewis et al., 1980); ASH response is not affected by levamisole treatment (Tanimoto et al., 2017).

464 For odor stimulation, we delivered a mixture of 2 -nonanone and air at a total of $8 \mathrm{~mm} / \mathrm{min}$ and created a temporal changing gradient of odor concentration by changing the ratio of 2 nonanone to air. We measured this gradient using a custom-made semiconductor sensor before and after performing the calcium imaging experiments on each day (Tanimoto and Kimura, 2021).

We divided the fluorescent signals of GCaMP3 and mCherry through a dual-wavelength measurement optical system, w-view (Hamamatsu Photonics), and captured their fluorescence images using an EM-CCD camera (ImagEM, Hamamatsu Photonics) at a sampling rate of 10 Hz. After subtracting the background, we extracted the fluorescence intensity of the cell body using Image $(\mathrm{NIH})$ and used their ratio (GCaMP/mCherry) as the data. 
477 Calcium imaging of ASH was performed using OSB2 under the following three conditions.

478 (1) Naive: the animals were cultivated on $6 \mathrm{~cm}$ NGM plates with OP50 provided as food, and

479 were then washed briefly with NGM buffer and measured; (2) Preexp: the animals were

480 preexposed to $0.6 \mu \mathrm{l}$ of $15 \%$ 2-nonanone (diluted in ethanol) for 1 hour on a $6 \mathrm{~cm}$ NGM plate

481 without food; and (3) Mock-treated: the animals were preexposed to ethanol in the same way

482 as the preexp condition. The mock-treated condition is a control that shows that starvation

483 itself does not affect the response to 2-nonanone.

484

485 Data analysis and statistical analysis.

486 In the calcium imaging experiments, data were obtained two to three days in each condition.

487 Experimental conditions, such as strains, odor stimuli and conditioning of the animals, were

488 randomly set for each day. After the data acquisition, animals with too weak intensity of basal

489 mCherry or GCaMP3 fluorescence for tracking were excluded. To exclude noise, frames in

490 which the GCaMP/mCherry ratio was in the top $1 \%$ and bottom $1 \%$ of the total were

491 removed. Statistical analyses were performed using the Kruskal-Wallis test with a post-hoc

492 Steel-Dwass test using R (The R Project). In the figure, * indicates $p<0.05$, ** indicates $p<$

4930.01 , and $* * *$ indicates $p<0.001$. Scatter plots represent median \pm quartiles and other graphs

494 represent the mean \pm standard error. We chose the sample size based on a large scale

495 behavioral analysis of C. elegans (Yemini et al., 2013).

496

497 Mathematical modeling of AWB-like responses by leaky integration.

498 Mathematical modeling of AWB-like responses was described in our previous work

499 (Tanimoto et al., 2017). In brief, the activity of AWB neurons, which responds to decreases in

500 2-nonanone concentration, is well fitted with the following leaky integrator equation:

501

$$
\frac{d X(t)}{d t}=I-\frac{1}{\tau}\left(X(t)-X_{e}\right)
$$




$$
I=-k \frac{d C(t)}{d t}
$$

503 where $X(t)$ is the measured fluorescence signal of the neuron (GCaMP/mCherry), $I$ is the

504 input, $X_{e}$ is the basal calcium level in the steady state, $k$ and $1 / \tau$ are the parameters, and $C(t)$ is

505 the measured odor concentration. The model parameters $k, 1 / \tau$, and $X_{e}$ were determined by the

506 least-squares method (Excel solver). This Eq. (1) indicates that the neuronal response $X(t)$

507 increases according to the input (negative $d C(t) / d t$ ) and reduces ("leaks") according to the $X(t)$

508 itself and returns to the steady state $X_{e}$. For example, when an animal experiences a constant

509 decrease in odor concentration (e.g., Figure 1-figure supplement 2C), the constant decrease

510 is transformed to a constant depolarization, and it causes constant calcium influx via L-type

511 VGCC EGL-19, and the calcium concentration reduces according to its total amount possibly

512 via leak channel(s) (Tanimoto et al., 2017). Since AWB neurons respond to decreases in odor

513 concentration, zero is substituted to $d C(t) / d t$ when $d C(t) / d t$ is positive in Eq. (1). This AWB

514 activity was essentially abolished by treatment of animals with Nemadipine-A (NemA), the

515 antagonist of C. elegans L-type VGCC (Kwok et al., 2006), indicating that the AWB activity

516 is mainly mediated by the L-type VGCC homolog EGL-19.

518 In NemA-treated animals, ASH neurons are rapidly activated at the beginning of the odor-

519 increasing phase but soon inactivated gradually even though the odor concentration kept

520 increasing (red dotted line in Figure 1-figure supplement 2D). When we assumed that the

521 NemA-treatment suppressed AWB-like leaky integration activity (yellow dotted line in Figure

522 1-figure supplement 2D), the sum of the remaining and the suppressed activity (blue solid

523 line in Figure 1-figure supplement 2D) nicely resembled the measured ASH activity (black

524 solid line in Figure 1-figure supplement 2D). We call the transient and gradual activities the

525 fast and slow components, respectively (Tanimoto et al., 2017). 
528 We extended the previous model Eq. (1) by introducing the second-order time differential of

529 input $C$ representing the fast component:

$$
\frac{d X(t)}{d t}=k_{1} \frac{d C(t)}{d t}+k_{2} \frac{d^{2} C(t)}{d t^{2}}-\frac{1}{\tau}\left(X(t)-X_{e}\right)
$$

where $X(t)$ is the measured ASH signal, $X_{e}$ is the basal calcium level, and $k_{1}, k_{2}$ and $1 / \tau$ are parameters for the first- and second-order time differential and leaky part, respectively. The parameters $k_{1}, k_{2}, 1 / \tau$, and $X_{e}$ were determined by the least-squares method for each odor stimulus. $d C(t) / d t$ and $d^{2} C(t) / d t^{2}$ were calculated using the central difference scheme. Since ASH neurons respond to increases in odor concentration (Tanimoto et al., 2017), zero is substituted to $d C(t) / d t$ or $d^{2} C(t) / d t^{2}$ when $d C(t) / d t$ or $d^{2} C(t) / d t^{2}$ is negative in Eq. (2).

The first and second differential model Eq. (2) is further extended on the terms of the secondorder time-differential and $X_{e}$ :

$$
\frac{d X(t)}{d t}=k_{1} \frac{d C(t)}{d t}+\Phi\left(k_{2} \frac{d^{2} C(t)}{d t^{2}}\right)-\frac{1}{\tau}\left(X(t)-X_{e}(t)\right)
$$

541 where $\Phi(x)$ is the saturation function as the following equation:

$$
\Phi(x)=\left\{\begin{array}{l}
0.14, \text { if } x>0.14 \\
x, \text { if } x \leq 0.14
\end{array}\right.
$$

543 This saturation function is introduced to prevent the parameters from becoming too large

544 when the increase in odor concentration approaches zero. 0.14 was arbitrary determined.

$545 X_{e}(t)$ changes over time as follows:

546

$$
X_{e}(t)= \begin{cases}b_{1} & \text {,if } t<t_{1} \\ \frac{b_{2}-b_{1}}{t_{2}-t_{1}} t+\frac{t_{2} b_{1}-t_{1} b_{2}}{t_{2}-t_{1}}, & \text { if } t_{1} \leq t \leq t_{2} \\ b_{2} & \text {,if } t_{2}<t\end{cases}
$$


where $t_{1}$ is the time at the start of the first odor increase phase, and $t_{2}$ is the time at the end of

548 the first odor increase phase. The parameters $b_{1}$ and $b_{2}$ were determined by the least-squares

549 method with the actual ASH response during 30 seconds until 10 seconds before the start of

550 the first odor increase phase $\left(t_{1}\right)$ and 1 minute after the first odor stimulus $\left(t_{2}\right)$, respectively.

551 Eq. (5) was introduced because the basal calcium level changed after the first odor stimulus.

552

553 In terms of parameters in Eq. (3), we plotted the relationships among parameters $\left(k_{1}, k_{2}\right.$, and

$5541 / \tau$ ) and various aspects of odor inputs (average, maximum, or accumulation of $C, d C / d t$, and

$\left.555 d^{2} C / d t^{2}\right)$ and fitted the relationships to the logarithmic function $(\mathrm{y}=f \ln (\mathrm{x})+g)$ of the odor

556 inputs (Excel solver) (Figure 3A and Figure 3-figure supplement 2); fitting of $k_{1}$ and $1 / \tau$ to

557 linear functions $(\mathrm{y}=h \mathrm{x}+i)$ of the odor inputs was worsened (Table 3$)$. We chose the aspect

558 of odor input with the smallest or the second smallest residual sum of squares, and the

559 logarithmic function of our chosen aspect for $k_{l}$ and $1 / \tau$ were similar between naive, mock-

560 treated and preexposed conditions. Thus we further optimized $f$ and $g$ manually such that they

561 were the same for all three conditions.

563 As a lower threshold for $1 / \tau$, the following function is introduced:

$$
\frac{1}{\tau}=\left\{\begin{array}{l}
0.02 \ln (x)+0.11, \text { if } x>0.1 \\
0.02 \ln (0.1)+0.11, \text { if } x \leq 0.1
\end{array}\right.
$$

where $x$ denotes $d^{2} C(t) / d t^{2}$ (Figure 3A). This function is introduced to prevent $1 / \tau$ from

566 becoming too small when the increase in odor concentration approaches zero. 0.1 was

567 arbitrary determined.

\section{Evaluation of the mathematical model.}

570 The Bayesian information criterion (BIC) (Schwarz, 1978) was used to assess the fit of the 
data using a mathematical model. The smaller the BIC value, the better is the model fit. In BIC, the goodness of fit for the model, including a penalty term to prevent overfitting, is given by the following equation:

$$
\mathrm{BIC}=N \ln \left(\frac{R S S}{N}\right)+M \ln (N)
$$

where $N$ is the number of frames used for the fitting, $R S S$ is the sum of the squared residuals between the model and the actual response, and $M$ is the number of free parameters used in the model. $M=2$ for the original simple time-differential model (Figure 2-figure supplement

1) because of $k$ and $X_{e}, M=3$ for the model with the leaky integration of only first- or secondorder time-differential (Figure 2-figure supplement 2 and 3) because of $k_{1}$ or $k_{2}$ and $1 / \tau$ and $X_{e}, M=4$ for the first and second differential model (Figure 2B) because of $k_{1}, k_{2}, 1 / \tau$ and $X_{e}$, and $M=12$ for the first and second differential model with stimulus-dependent parameters (Figure 3B) because there were two parameters each in the stimulus-dependent parameters $k_{l}$, $k_{2}$, and $1 / \tau$, four parameters in $X_{e}(t)$, one parameter in $\Phi(x)$, and one parameter in lower

584 threshold for $1 / \tau$.

\section{Behavioral analysis.}

587 Trajectories obtained by a high-resolution USB camera (DMK72AUC02; The Imaging

588 Source, United States) during 2-nonanone avoidance behavior were clustered using the 589 previously reported STEFTER method (Yamazaki et al., 2019). In brief, clustering was

590 performed using variances of temporal changes in bearing, and the cluster with the smallest variances of temporal changes in bearing was classified as "run", while the other clusters were classified as "pirouette" categories. This classification of "run" and "pirouette" was more than $90 \%$ consistent with the previous one based on the durations of straight migration

594 (Yamazoe-Umemoto et al., 2015). Then, the change in the odor concentration sensed during 595 the run was calculated according to an odor gradient model based on the measured odor 
concentrations (Yamazoe-Umemoto et al., 2015). The behavioral data used in this study have already been analyzed and published previously (Yamazaki et al., 2019).

\section{ACKNOWLEDGEMENTS}

We thank Yuki Tsukada, Yuishi Iwasaki, Sakiko Shiga, Masahiro Ueda, and the other Kimura laboratory members for their valuable advice, comments and technical assistance for this study. Nematode strains were provided by the Caenorhabditis Genetics Center (funded by the NIH Office of Research Infrastructure Programs P40 OD010440).

\section{FUNDING}

This study was supported by Japan Society for the Promotion of Science (KAKENHI JP16H06545, 21H02533 to K.D.K., 17H05968 and 19H04925 to Y.Iwatani), a program for Leading Graduate Schools entitled 'Interdisciplinary graduate school program for systematic understanding of health and disease' (to Y.T., S.J.Y. and Y.E.), Grant-in-Aid for Research in Nagoya City University (48, 1912011, 1921102, 2121101), Toyoaki Scholarship Foundation, and RIKEN Center for Advanced Intelligence Project (to K.D.K).

\section{COMPETING INTERESTS}

The authors declare no competing interests.

\section{REFERENCE}

Andersen RA, Essick GK, Siegel RM. 1985. Encoding of Spatial location by Posterior Parietal Neurons. Science 230:456-458. doi:10.1126/science.4048942.

Anderson CT, Kumar M, Xiong S, Tzounopoulos T. 2017. Cell-specific gain modulation by synaptically released zinc in cortical circuits of audition. Elife 6:1-20. doi:10.7554/eLife.29893

Azimi Z, Barzan R, Spoida K, Surdin T, Wollenweber P, Mark MD, Herlitze S, Jancke D. 

2020. Separable gain control of ongoing and evoked activity in the visual cortex by serotonergic input. Elife 9:1-29. doi:10.7554/eLife.53552

Bargmann CI. 2006. Chemosensation in C. elegans. WormBook 1-29. doi:10.1895/wormbook.1.123.1

Bargmann CI, Hartwieg E, Horvitz HR. 1993. Odorant-selective genes and neurons mediate olfaction in C. elegans. Cell 74:515-527. doi:10.1016/0092-8674(93)80053-H

Brenner S. 1974. The genetics of Caenorhabditis elegans. Genetics 77:71-94. doi:10.1093/genetics/77.1.71.

Cao Y, Pahlberg J, Sarria I, Kamasawa N, Sampath AP, Martemyanov KA. 2012. Regulators of G protein signaling RGS7 and RGS11 determine the onset of the light response in on bipolar neurons. Proc Natl Acad Sci U S A 109:7905-7910. doi:10.1073/pnas.1202332109

Carew TJ, Castellucci VF, Kandel ER. 1971. An analysis of dishabituation and sensitization of the gill-withdrawal reflex in aplysia. Int J Neurosci 2:79-98. doi:10.3109/00207457109146995

Chance FS, Abbott LF, Reyes AD. 2002. Gain modulation from background synaptic input. Neuron 35:773-782. doi:10.1016/S0896-6273(02)00820-6

Chew YL, Tanizawa Y, Cho Y, Zhao B, Yu AJ, Ardiel EL, Rabinowitch I, Bai J, Rankin CH, Lu H, Beets I, Schafer WR. 2018. An Afferent Neuropeptide System Transmits Mechanosensory Signals Triggering Sensitization and Arousal in C. elegans. Neuron 99:1233-1246.e6. doi:10.1016/j.neuron.2018.08.003

Colbert HA, Smith TL, Bargmann CI. 1997. OSM-9, a novel protein with structural similarity to channels, is required for olfaction, mechanosensation, and olfactory adaptation in Caenorhabditis elegans. J Neurosci 17:8259-8269. doi:10.1523/jneurosci.17-2108259.1997

De Bono M, Maricq AV. 2005. Neuronal substrates of complex behaviors in C. elegans. Annu Rev Neurosci 28:451-501. doi:10.1146/annurev.neuro.27.070203.144259

Dragoi V, Sharma J, Sur M. 2000. Adaptation-induced plasticity of orientation tuning in adult visual cortex. Neuron 28:287-298. doi:10.1016/S0896-6273(00)00103-3

Ezcurra M, Walker DS, Beets I, Swoboda P, Schafer WR. 2016. Neuropeptidergic signaling and active feeding state inhibit nociception in Caenorhabditis elegans. $J$ Neurosci 36:3157-3169. doi:10.1523/JNEUROSCI.1128-15.2016

Ferkey DM, Hyde R, Haspel G, Dionne HM, Hess HA, Suzuki H, Schafer WR, Koelle MR, Hart AC. 2007. C. elegans G Protein Regulator RGS-3 Controls Sensitivity to Sensory Stimuli. Neuron 53:39-52. doi:10.1016/j.neuron.2006.11.015

Ferkey DM, Sengupta P, L'Etoile ND. 2021. Chemosensory signal transduction in Caenorhabditis elegans. Genetics 217. doi:10.1093/GENETICS/IYAB004

Huang J, Zhou H, Mahavadi S, Sriwai W, Murthy KS. 2007. Inhibition of Gaq-dependent 

PLC- $\beta 1$ activity by PKG and PKA is mediated by phosphorylation of RGS4 and GRK2. Am J Physiol - Cell Physiol 292:200-208. doi:10.1152/ajpcell.00103.2006

Ikeda M, Matsumoto H, Izquierdo EJ. 2021. Persistent thermal input controls steering behavior in Caenorhabditis elegans. PLoS Comput Biol 17. doi:10.1371/JOURNAL.PCBI.1007916

Itskovits E, Ruach R, Kazakov A, Zaslaver A. 2018. Concerted pulsatile and graded neural dynamics enables efficient chemotaxis in C. elegans. Nat Commun 9. doi:10.1038/s41467-018-05151-2

Kaplan JM, Horvitz HR. 1993. A dual mechanosensory and chemosensory neuron in Caenorhabditis elegans. Proc Natl Acad Sci U S A 90:2227-2231. doi:10.1073/pnas.90.6.2227

Kato S, Xu Y, Cho CE, Abbott LF, Bargmann CI. 2014. Temporal Responses of C.elegans Chemosensory Neurons Are Preserved in Behavioral Dynamics. Neuron 81:616-628. doi:10.1016/j.neuron.2013.11.020

Kimura KD, Fujita K, Katsura I. 2010. Enhancement of odor avoidance regulated by dopamine signaling in Caenorhabditis elegans. J Neurosci 30:16365-16375. doi:10.1523/JNEUROSCI.6023-09.2010

Krzyzanowski MC, Brueggemann C, Ezak MJ, Wood JF, Michaels KL, Jackson CA, Juang BT, Collins KD, Yu MC, L'Etoile ND, Ferkey DM. 2013. The C. elegans cGMPDependent Protein Kinase EGL-4 Regulates Nociceptive Behavioral Sensitivity. PLoS Genet 9. doi:10.1371/journal.pgen.1003619

Kuhara A, Inada H, Katsura I, Mori I. 2002. Negative regulation and gain control of sensory neurons by the C. elegans calcineurin TAX-6. Neuron 33:751-763. doi:10.1016/S08966273(02)00607-4

Kwok TCY, Ricker N, Fraser R, Chan AW, Burns A, Stanley EF, McCourt P, Cutler SR, Roy PJ. 2006. A small-molecule screen in C. elegans yields a new calcium channel antagonist. Nature 441:91-95. doi:10.1038/nature04657

L'Etoile ND, Coburn CM, Eastham J, Kistler A, Gallegos G, Bargmann CI. 2002. The cyclic GMP-dependent protein kinase EGL-4 regulates olfactory adaptation in C. elegans. Neuron 36:1079-1089. doi:10.1016/S0896-6273(02)01066-8

Labows JN, McGinley KJ, Webster GF, Leyden JJ. 1980. Headspace analysis of volatile metabolites of Pseudomonas aeruginosa and related species by gas chromatographymass spectrometry. J Clin Microbiol 12:521-526. doi:10.1128/jcm.12.4.521-526.1980

Lainé V, Frøkjær-Jensen C, Couchoux H, Jospin M. 2011. The $\alpha 1$ subunit EGL-19, the $\alpha 2 / \delta$ subunit UNC-36, and the $\beta$ subunit CCB-1 underlie voltage-dependent calcium currents in Caenorhabditis elegans striated muscle. J Biol Chem 286:36180-36187. doi:10.1074/jbc.M111.256149

Larsch J, Flavell SW, Liu Q, Gordus A, Albrecht DR, Bargmann CI. 2015. A Circuit for Gradient Climbing in C. elegans Chemotaxis. Cell Rep 12:1748-1760. doi:10.1016/j.celrep.2015.08.032 
701

702

703

704

705

706

707

708

709

710

711

712

713

714

715

716

717

718

719

720

721

722

723

724

725

726

727

728

729

730

731

732

733

734

735

736

737

738

Lewis JA, Wu CH, Berg H, Levine JH. 1980. The genetics of levamisole resistance in the nematode Caenorhabditis elegans. Genetics 95:905-928. doi:10.1093/genetics/95.4.905

Lur G, Higley MJ. 2015. Glutamate Receptor Modulation Is Restricted to Synaptic Microdomains. Cell Rep 12:326-334. doi:10.1016/j.celrep.2015.06.029

Nowycky MC, Fox AP, Tsien RW. 1985. Three types of neuronal calcium channel with different calcium agonist sensitivity. Nature 316:440-3. doi:10.1038/316440a0

Ohzawa I, Sclar G, Freeman RD. 1982. Contrast gain control in the cat visual cortex. Nature 298:266-268. doi:10.1038/298266a0

Olsen SR, Wilson RI. 2008. Lateral presynaptic inhibition mediates gain control in an olfactory circuit. Nature 452:956-960. doi:10.1038/nature06864

Pierce-Shimomura JT, Morse TM, Lockery SR. 1999. The fundamental role of pirouettes in Caenorhabditis elegans chemotaxis. J Neurosci 19:9557-9569. doi:10.1523/jneurosci.19-21-09557.1999

Priebe NJ, Ferster D. 2002. A new mechanism for neuronal gain control (or how the gain in brains has mainly been explained). Neuron 35:602-604. doi:10.1016/S08966273(02)00829-2

Rall W. 1967. Distinguishing Theoretical Synaptic Potentials Computed for Different SomaDendritic Distributions of Synaptic. J Neurophysiol 5:1138-68. doi:10.1152/jn.1967.30.5.1138

Root CM, Masuyama K, Green DS, Enell LE, Nässel DR, Lee CH, Wang JW. 2008. A Presynaptic Gain Control Mechanism Fine-Tunes Olfactory Behavior. Neuron 59:311321. doi:10.1016/j.neuron.2008.07.003

Saro G, Lia AS, Thapliyal S, Marques F, Busch KE, Glauser DA. 2020. Specific Ion Channels Control Sensory Gain, Sensitivity, and Kinetics in a Tonic Thermonociceptor. Cell Rep 30:397-408.e4. doi:10.1016/j.celrep.2019.12.029

Sasakura H, Mori I. 2013. Behavioral plasticity, learning, and memory in C. elegans. Curr Opin Neurobiol 23:92-99. doi:10.1016/j.conb.2012.09.005

Schwarz G. 1978. Estimating the dimension of a model. Ann Stat 6:461-464. doi:10.1214/aos/1176344136

Shaner NC, Campbell RE, Steinbach PA, Giepmans BNG, Palmer AE, Tsien RY. 2004. Improved monomeric red, orange and yellow fluorescent proteins derived from Discosoma sp. red fluorescent protein. Nat Biotechnol 22:1567-1572. doi: $10.1038 / \mathrm{nbt} 1037$

Shapley R, Enroth-Cugell C. 1984. Visual Adaptation and Retinal Gain Controls. Eng Sci 3:263-346. doi:10.1016/0278-4327(84)90011-7.

Steger KA, Shtonda BB, Thacker C, Snutch TP, Avery L. 2005. The C. elegans T-type calcium channel CCA-1 boosts neuromuscular transmission. J Exp Biol 208:2191-2203. doi: $10.1242 /$ jeb.01616 
Tan MW, Ausubel FM. 2000. Caenorhabditis elegans: A model genetic host to study Pseudomonas aeruginosa pathogenesis. Curr Opin Microbiol 3:29-34. doi:10.1016/S1369-5274(99)00047-8

Tan MW, Mahajan-Miklos S, Ausubel FM. 1999. Killing of Caenorhabditis elegans by Pseudomonas aeruginosa used to model mammalian bacterial pathogenesis. Proc Natl Acad Sci U S A 96:715-720. doi:10.1073/pnas.96.2.715

Tanimoto Y, Kimura KD. 2021. Calcium Imaging of Neuronal Activity under Gradually Changing Odor Stimulation in Caenorhabditis elegans. Bio-Protocol 11:1-23. doi: $10.21769 /$ bioprotoc. 3866

Tanimoto Y, Yamazoe-Umemoto A, Fujita K, Kawazoe Y, Miyanishi Y, Yamazaki SJ, Fei X, Busch KE, Gengyo-Ando K, Nakai J, Iino Y, Iwasaki Y, Hashimoto K, Kimura KD. 2017. Calcium dynamics regulating the timing of decision-making in C. Elegans. Elife 6:1-30. doi:10.7554/eLife.21629

Tian L, Hires SA, Mao T, Huber D, Chiappe ME, Chalasani SH, Petreanu L, Akerboom J, McKinney SA, Schreiter ER, Bargmann CI, Jayaraman V, Svoboda K, Looger LL. 2009. Imaging neural activity in worms, flies and mice with improved GCaMP calcium indicators. Nat Methods 6:875-881. doi:10.1038/nmeth.1398

Tobin DM, Madsen DM, Kahn-Kirby A, Peckol EL, Moulder G, Barstead R, Maricq A V., Bargmann CI. 2002. Combinatorial expression of TRPV channel proteins defines their sensory functions and subcellular localization in C. elegans neurons. Neuron 35:307318. doi:10.1016/S0896-6273(02)00757-2

Ulanovsky N, Las L, Nelken I. 2003. Processing of low-probability sounds by cortical neurons. Nat Neurosci 6:391-398. doi:10.1038/nn1032

Woolf CJ, Ma Q. 2007. Nociceptors-Noxious Stimulus Detectors. Neuron 55:353-364. doi:10.1016/j.neuron.2007.07.016

Yamazaki SJ, Ohara K, Ito K, Kokubun N, Kitanishi T, Takaichi D, Yamada Y, Ikejiri Y, Hiramatsu F, Fujita K, Tanimoto Y, Yamazoe-Umemoto A, Hashimoto K, Sato K, Yoda K, Takahashi A, Ishikawa Y, Kamikouchi A, Hiryu S, Maekawa T, Kimura KD. 2019. SteftR: A hybrid versatile method for state estimation and feature extraction from the trajectory of animal behavior. Front Neurosci 13. doi:10.3389/fnins.2019.00626

Yamazoe-Umemoto A, Fujita K, Iino Y, Iwasaki Y, Kimura KD. 2015. Modulation of different behavioral components by neuropeptide and dopamine signalings in nonassociative odor learning of Caenorhabditis elegans. Neurosci Res 99:22-33. doi:10.1016/j.neures.2015.05.009

Yeh E, Ng S, Zhang M, Bouhours M, Wang Y, Wang M, Hung W, Aoyagi K, MelnikMartinez K, Li M, Liu F, Schafer WR, Zhen M. 2008. A putative cation channel, NCA1, and a novel protein, UNC-80, transmit neuronal activity in C. elegans. PLoS Biol 6:0552-0567. doi:10.1371/journal.pbio.0060055

Yemini E, Jucikas T, Grundy LJ, Brown AEX, Schafer WR. 2013. A database of Caenorhabditis elegans behavioral phenotypes. Nat Methods 10:877-879. doi:10.1038/nmeth. 2560 


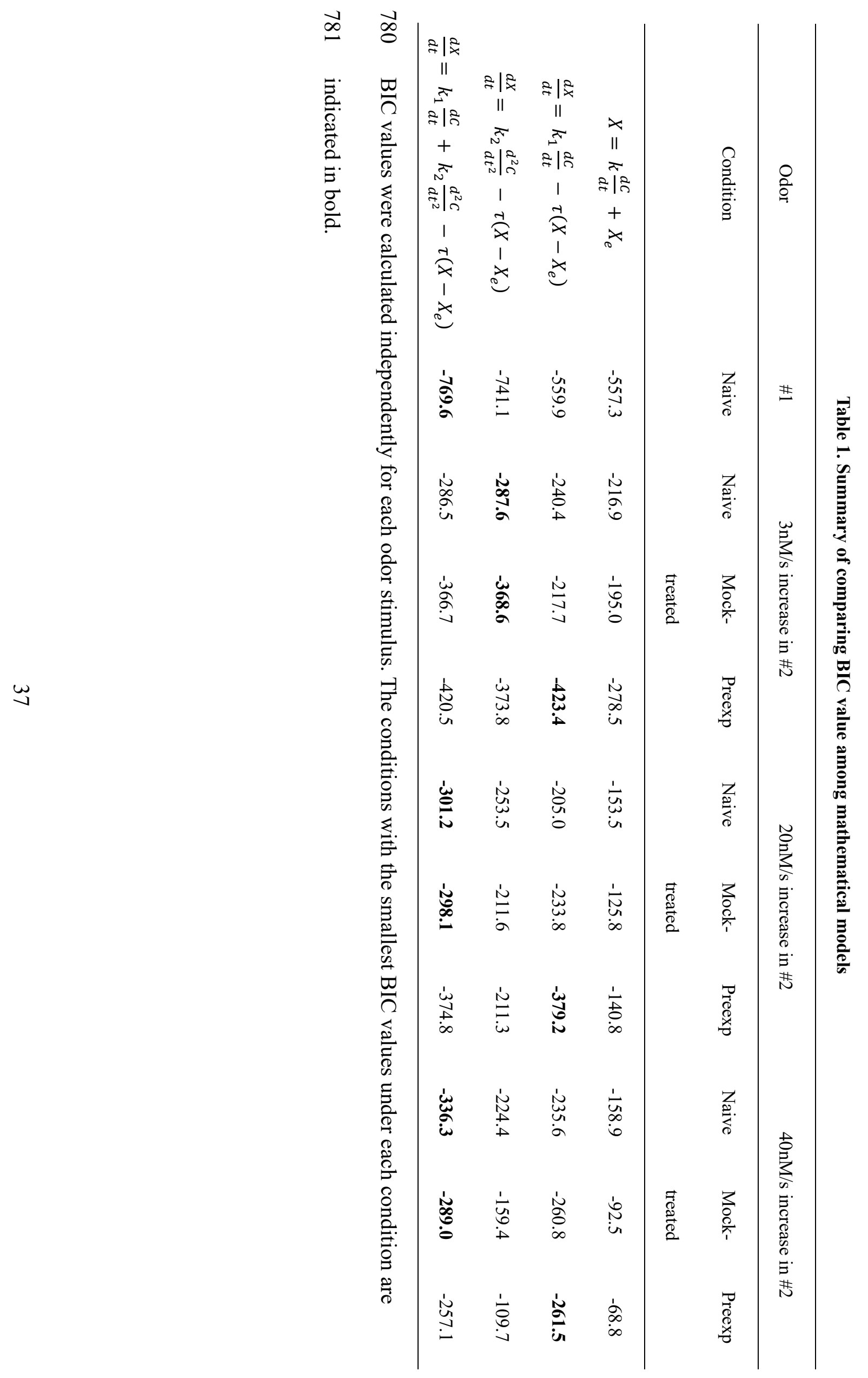




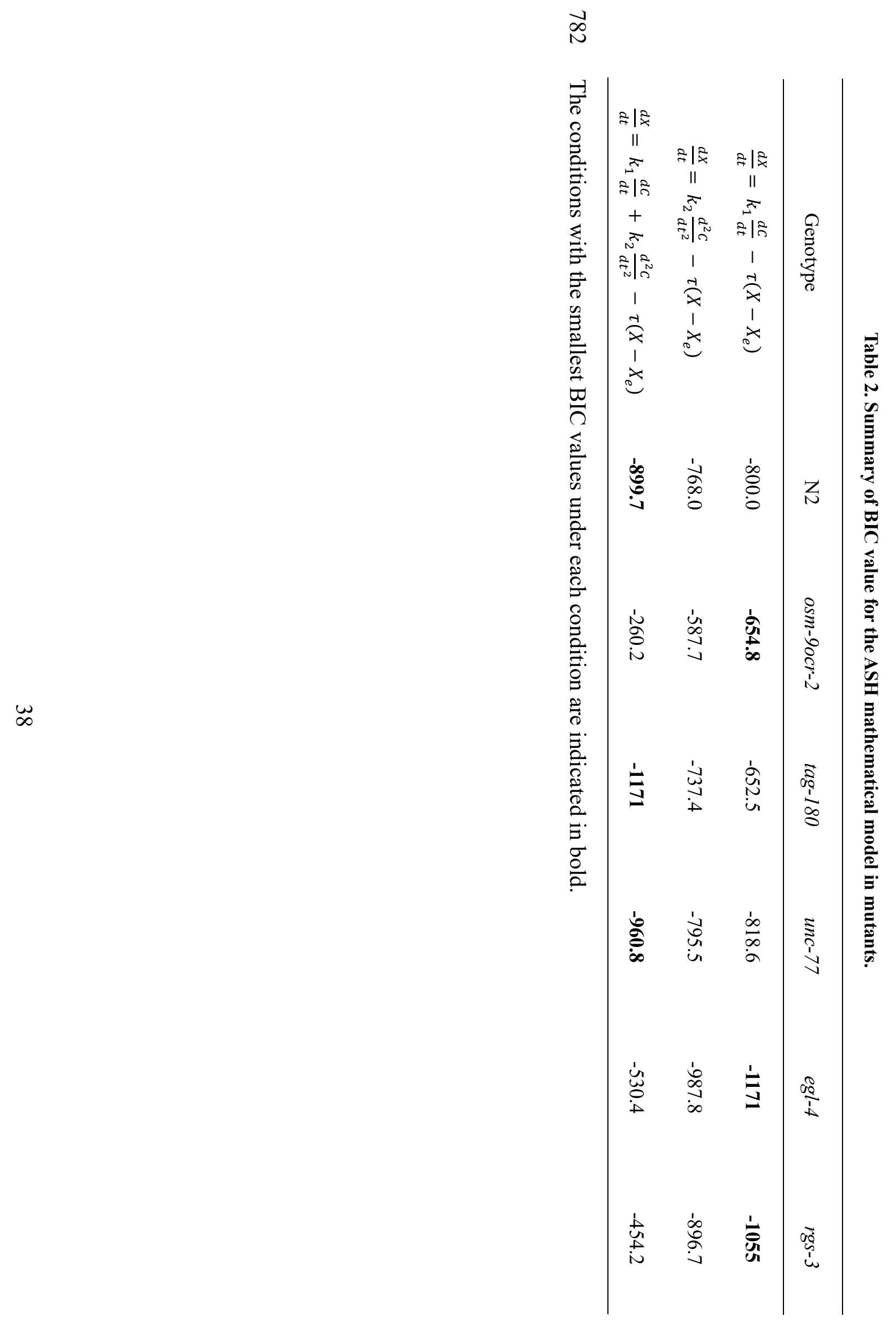


$\stackrel{\infty}{\infty}$

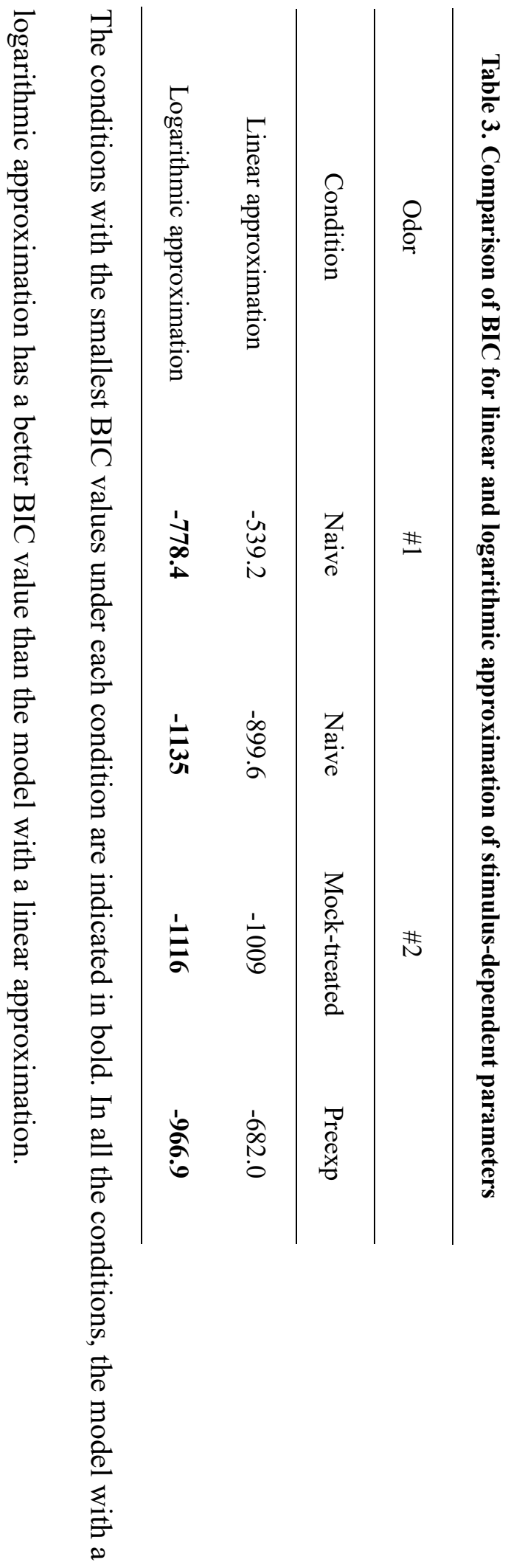

